\title{
Long-time Dynamics and Optimal Control of a Diffuse Interface Model for Tumor Growth
}

\author{
Cecilia Cavaterra * ${ }^{*} \quad$ Elisabetta Rocca ${ }^{\dagger} \quad \mathrm{Hao} \mathrm{Wu}^{\ddagger}$
}

January 23, 2019

\begin{abstract}
We investigate the long-time dynamics and optimal control problem of a diffuse interface model that describes the growth of a tumor in presence of a nutrient and surrounded by host tissues. The state system consists of a Cahn-Hilliard type equation for the tumor cell fraction and a reaction-diffusion equation for the nutrient. The possible medication that serves to eliminate tumor cells is in terms of drugs and is introduced into the system through the nutrient. In this setting, the control variable acts as an external source in the nutrient equation. First, we consider the problem of "long-time treatment" under a suitable given source and prove the convergence of any global solution to a single equilibrium as $t \rightarrow+\infty$. Then we consider the "finite-time treatment" of a tumor, which corresponds to an optimal control problem. Here we also allow the objective cost functional to depend on a free time variable, which represents the unknown treatment time to be optimized. We prove the existence of an optimal control and obtain first order necessary optimality conditions for both the drug concentration and the treatment time. One of the main aim of the control problem is to realize in the best possible way a desired final distribution of the tumor cells, which is expressed by the target function $\phi_{\Omega}$. By establishing the Lyapunov stability of certain equilibria of the state system (without external source), we see that $\phi_{\Omega}$ can be taken as a stable configuration, so that the tumor will not grow again once the finite-time treatment is completed.
\end{abstract}

Keywords: Tumor growth, Cahn-Hilliard equation, reaction-diffusion equation, optimal control, long-time behavior, Lyapunov stability.

AMS Subject Classification: 35K61, 49J20, 49K20, 92C50, 97M60.

\section{Introduction}

Modeling tumor growth dynamics has recently become a major issue in applied mathematics (see, for instance, [19,43, cf. also [2,49]). Roughly speaking, the models can be divided into two broad categories: continuum models and discrete or cellular automata models (however, see, e.g., [19, Chap.7] for some hybrid continuum-discrete models). Concerning the former ones and

\footnotetext{
*Dipartimento di Matematica, Università degli Studi di Milano, Via Saldini 50, 20133 Milano, Italy and Istituto di Matematica Applicata e Tecnologie Informatiche "Enrico Magenes", CNR, Via Ferrata 1, 27100 Pavia, Italy. cecilia.cavaterra@unimi.it

${ }^{\dagger}$ Dipartimento di Matematica, Università degli Studi di Pavia, Via Ferrata 5, 27100 Pavia, Italy and Istituto di Matematica Applicata e Tecnologie Informatiche "Enrico Magenes", CNR, Via Ferrata 1, 27100 Pavia, Italy. elisabetta.rocca@unipv.it

${ }^{\ddagger}$ School of Mathematical Sciences; Key Laboratory of Mathematics for Nonlinear Sciences (Fudan University), Ministry of Education; Shanghai Key Laboratory for Contemporary Applied Mathematics, Fudan University, Han Dan Road 220, 200433 Shanghai, China. haowufd@fudan.edu.cn
} 
in particular within the framework of diffuse interface models, for a young tumor, before the development of quiescent cells, the resulting systems often consist of a Cahn-Hilliard type equation (cf. [7) for the tumor cell fraction coupled with an advection-reaction-diffusion equation for the nutrient concentration (e.g., oxygen) [11 15, 24, 29 31, 35, 37, 47, 50. More sophisticated models taking into account multiphase tumors or also accounting for the macroscopic cell velocities that usually satisfy a generalized Darcy's (or Brinkman's) law have been recently studied, e.g., in $5,19,22,25,28,30,32,41,43,45$. Besides, numerical simulations of diffuse-interface models for tumor growth have been carried out in several papers (see, for instance, [19, Chap.8], [1], and references therein). Nonetheless, a rigorous mathematical analysis of the resulting systems of partial differential equations is still in its infancy. To the best of our knowledge, the first related papers are concerned with the so-called Cahn-Hilliard-Hele-Shaw system (see [44,56, 57]), in which the nutrient is neglected. Besides, there are recent contributions (see [11] and [24]) devoted to analyzing a diffuse interface model proposed in [35. and its approximations (see also [37, 58] and $[13[15]$ ).

Let $\Omega \subset \mathbb{R}^{d}(d=2,3)$ be a bounded domain with smooth boundary $\partial \Omega$ and let $\nu$ denote the outward unit normal to $\partial \Omega$. For $T \in(0,+\infty]$, we study the following coupled system of partial differential equations

$$
\begin{array}{ll}
\phi_{t}-\Delta \mu=P(\phi)(\sigma-\mu), & \text { in } \Omega \times(0, T), \\
\mu=-\Delta \phi+F^{\prime}(\phi), & \text { in } \Omega \times(0, T), \\
\sigma_{t}-\Delta \sigma=-P(\phi)(\sigma-\mu)+u, & \text { in } \Omega \times(0, T),
\end{array}
$$

subject to homogeneous Neumann boundary conditions

$$
\partial_{\nu} \phi=\partial_{\nu} \mu=\partial_{\nu} \sigma=0, \quad \text { on } \partial \Omega \times(0, T),
$$

and initial conditions

$$
\left.\phi\right|_{t=0}=\phi_{0}(x),\left.\quad \sigma\right|_{t=0}=\sigma_{0}(x), \quad \text { in } \Omega .
$$

System (1.1) - (1.3) is an approximation of the model proposed in [35. The macroscopic velocities of cells are set to zero for the sake of simplicity. The state variables are reduced to the tumor cell fraction $\phi$ and the nutrient concentration $\sigma$. Typically, $\phi \simeq 1$ and $\phi \simeq-1$ represent the tumor phase and the healthy tissue phase respectively, while $\sigma \simeq 1$ and $\sigma \simeq 0$ indicate in a nutrient-rich or nutrient-poor extracellular water phase. The unknown $\mu$ stands for the related chemical potential and the function $F$ is typically a double-well potential with equal minima at $\phi= \pm 1$ (cf. [1] and references therein for different possible choices of $F$ ). $P$ denotes a suitable proliferation function, which is in general a nonnegative and regular function of $\phi$. The function $u$ serves as an external source in the equation for $\sigma$ and can be interpreted as a medication (or a nutrient supply).

In [24], the system (1.1)-(1.5) with $u=0$ was rigorously analyzed concerning wellposedness, regularity and long-time behavior (in terms of the global attractor), while in the recent paper 47] the long-time behavior of solutions (in terms of attractors) has been studied for a different system introduced in [32. Let us indeed notice that, to the best of our knowledge, these are the only two contriobutions in the literature regarding the long-term dynamics of diffuse interface models for tumor growth. While, in [11,13,14 various viscous approximations of the above system have been studied analytically. Later, for a fixed final time $T>0$, a distributed optimal control problem for system (1.1)-(1.5) was investigated in [15, in which the function $u$ was taken as the control. With a simplified cost function of standard tracking type that only involves the phase function $\phi$ and the control $u$, the authors of [15] first prove the existence of an optimal control and, moreover, they show that the control-to-state operator is Fréchet differentiable between 
appropriate Banach spaces and derive the first-order necessary optimality conditions in terms of a variational inequality involving the adjoint state variables.

We note that the choice of reactive terms in equations (1.1) and (1.3) is motivated by the linear phenomenological constitutive laws for chemical reactions 35. As a consequence, the system (1.1)-(1.5) turns out to be thermodynamically consistent. In particular, when $u=0$ the unknown pair $(\phi, \sigma)$ is a dissipative gradient flow for the total free energy (see [35, Section 3], see also [37]):

$$
\mathcal{E}(\phi, \sigma)=\int_{\Omega}\left[\frac{1}{2}|\nabla \phi|^{2}+F(\phi)\right] \mathrm{d} x+\frac{1}{2} \int_{\Omega} \sigma^{2} \mathrm{~d} x
$$

Moreover generally, under the presence of the external source $u$, we observe that any smooth solution $(\phi, \sigma)$ to problem (1.1) - (1.5) satisfies the following energy identity:

$$
\frac{\mathrm{d}}{\mathrm{d} t} \mathcal{E}(\phi, \sigma)+\int_{\Omega}\left[|\nabla \mu|^{2}+|\nabla \sigma|^{2}+P(\phi)(\mu-\sigma)^{2}\right] \mathrm{d} x=\int_{\Omega} u \sigma \mathrm{d} x, \quad \forall t>0,
$$

which motives the twofold aim of the present contribution.

1. Long-time treatment of medication. For a suitably given external source $u$, we study the long-term dynamics of problem (1.1)-(1.5). We prove that any global weak solution will converge to a single equilibrium as $t \rightarrow+\infty$ and provide an estimate on the convergence rate. The related main result is stated in Section 3 (see Theorem 3.1).

In this direction, our result indicates that after certain medication (or even without medication, i.e., $u=0$ ), the tumor will eventually grow to a steady state as time evolves. However, since the potential function $F$ is nonconvex due to its double-well structure, problem (1.1)-(1.5) may admit infinite many steady states so that for the moment one cannot identify which exactly the unique asymptotic limit as $t \rightarrow+\infty$ will be.

2. Finite-time treatment of medication. We investigate a more general distributed optimal control problem (cf. [15]), where we allow the objective cost functional to depend also on a free time variable, representing the unknown treatment time to be optimized. More precisely, denoting by $T \in(0,+\infty)$ a fixed maximal time in which the patient is allowed to undergo a medical treatment, we consider

(CP) Minimize the cost functional

$$
\begin{aligned}
\mathcal{J}(\phi, \sigma, u, \tau)= & \frac{\beta_{Q}}{2} \int_{0}^{\tau} \int_{\Omega}\left|\phi-\phi_{Q}\right|^{2} \mathrm{~d} x \mathrm{~d} t+\frac{\beta_{\Omega}}{2} \int_{\Omega}\left|\phi(\tau)-\phi_{\Omega}\right|^{2} \mathrm{~d} x \\
& +\frac{\alpha_{Q}}{2} \int_{0}^{\tau} \int_{\Omega}\left|\sigma-\sigma_{Q}\right|^{2} \mathrm{~d} x \mathrm{~d} t+\frac{\beta_{S}}{2} \int_{\Omega}(1+\phi(\tau)) \mathrm{d} x \\
& +\frac{\beta_{u}}{2} \int_{0}^{T} \int_{\Omega}|u|^{2} \mathrm{~d} x \mathrm{~d} t+\beta_{T} \tau,
\end{aligned}
$$

subject to the control constraint

$$
u \in \mathcal{U}_{\mathrm{ad}}:=\left\{u \in L^{\infty}(Q): u_{\min } \leq u \leq u_{\max } \text { a. e. in } Q\right\}, \quad \tau \in(0, T),
$$

and to the state system (1.1)-(1.5), where $Q:=\Omega \times(0, T)$.

Here, $\tau \in(0, T]$ represents the treatment time, $\phi_{Q}$ and $\sigma_{Q}$ represent a desired evolution for the tumor cells and for the nutrient, respectively, while $\phi_{\Omega}$ stands for desired final distribution of tumor cells. The first three terms of $\mathcal{J}$ are of standard tracking type, as often considered in the literature of parabolic optimal control, and the fourth term of $\mathcal{J}$ 
measures the size of the tumor at the end of the treatment. The fifth term penalizes large concentrations of the cytotoxic drugs, and the sixth term of $\mathcal{J}$ penalizes long treatment times. As it is presented in $\mathcal{J}$, a large value of $\left|\phi-\phi_{Q}\right|^{2}$ would mean that the patient suffers from the growth of the tumor, and a large value of $|u|^{2}$ would mean that the patient suffers from high toxicity of the drug. We shall prove the existence of an optimal control and derive the first-order necessary optimality conditions in terms of a variational inequality involving the adjoint state variables. The related main results are stated in Section 5 ,

The variable $\tau$ can be regarded as the necessary treatment time of one cycle, i.e., the amount of time the drug is applied to the patient before the period of rest, or the treatment time before surgery. After the treatment, the ideal situation will be either the tumor is ready for surgery or the tumor will be stable for all time without further medication (i.e., $u=0$ ). This goal can be realized by making different choices of the target function $\phi_{\Omega}$ in the above optimal control problem $(\mathbf{C P})$. For the former case, one can simply take $\phi_{\Omega}$ to be a configuration that is suitable for surgery. While for the later case, which is of more interest to us, we want to choose $\phi_{\Omega}$ as a "stable" configuration of the system, so that the tumor does not grow again once the treatment is complete. For this purpose, we prove that any local minimizer of the total free energy $\mathcal{E}$ is Lyapunov stable provided that $u=0$ (see Theorem 4.1). As a consequence, these local energy minimizers serve as possible candidates for the target function $\phi_{\Omega}$. Then after completing a successful medication, the tumor will remain close to the chosen stable configuration for all time.

Let us briefly describe some ingredients in the mathematical analysis. The study of long-time behavior of problem (1.1)-(1.5) is nontrivial, since the nonconvexity of the free energy $\mathcal{E}$ indicates that the set of steady states may have a rather complicated structure. For the single Cahn-Hilliard equation this difficulty can be overcome by employing the Łojasiewicz-Simon approach [53], see, for instance, [27, 42, 52, 59, 61. We also refer to [8, 23, 33, 40, 41, 60] and the references cited therein for further applications. A key property that plays an important role in the analysis of the Cahn-Hilliard equation is the conservation of mass, i.e., $\int_{\Omega} \phi(t) \mathrm{d} x=\int_{\Omega} \phi_{0} \mathrm{~d} x$ for $t \geq 0$. However, for our coupled system (1.1)-(1.5) this property no longer holds, which brings us new difficulties in analysis. Besides, quite different from the Cahn-Hilliard-Oono system considered in [46], in which the mass $\int_{\Omega} \phi(t) \mathrm{d} x$ is not preserved due to possible reactions, here in our case it is not obvious how to control the mass changing rate:

$$
\frac{\mathrm{d}}{\mathrm{d} t} \int_{\Omega} \phi \mathrm{d} x=\int_{\Omega} P(\phi)(\sigma-\mu) \mathrm{d} x .
$$

Similar problem happens to the nutrient as well, that is

$$
\frac{\mathrm{d}}{\mathrm{d} t} \int_{\Omega} \sigma \mathrm{d} x=-\int_{\Omega} P(\phi)(\sigma-\mu) \mathrm{d} x+\int_{\Omega} u \mathrm{~d} x .
$$

Nevertheless, by the special cancellation between those reactive terms in (1.1) and (1.3), we see that the total mass can be determined by the initial data and the external source:

$$
\int_{\Omega}(\phi(t)+\sigma(t)) \mathrm{d} x=\int_{\Omega}\left(\phi_{0}+\sigma_{0}\right) \mathrm{d} x+\int_{0}^{t} \int_{\Omega} u \mathrm{~d} x \mathrm{~d} \tau, \quad \forall t \geq 0 .
$$

This observation allows us to derive a suitable version of the Łojasiewicz-Simon type inequality in the sprit of [60,62] (see Appendix). On the other hand, we can control the mass changing rates of $\phi$ and $\sigma$ by using the extra energy dissipation related to reactive terms in the basic energy law (1.7), i.e., $\int_{\Omega} P(\phi)(\mu-\sigma)^{2} \mathrm{~d} x$. Based on the above mentioned special structure of the system, by introducing a new version of Łojasiewicz-Simon inequality (see Lemma 3.1), we are able to prove that every global weak solution $(\phi, \sigma)$ of problem (1.1)-(1.5) will converge to a 
certain single equilibrium $\left(\phi_{\infty}, \sigma_{\infty}\right)$ as $t \rightarrow+\infty$ and, moreover, we obtain a polynomial decay of the solution. Besides, a nontrivial application of the Łojasiewicz-Simon approach further leads to the Lyapunov stability of local minimizers of the free energy $\mathcal{E}$ (we only consider the zero external mass case $u=0$ for the sake of simplicity). To the best of our knowledge, the only contribution in the study of long-time behavior for problem (1.1)-(1.5) is given in [24] with $u=0$, where, however, the main focus is the existence of a global attractor (cf. also [47] for a different model). The novelty of our work is that we provide a first contribution in the literature on the uniqueness of asymptotic limit of global solutions as well as Lyapunov stability of steady states for the diffuse interface models on tumor growth with reaction terms like in (1.1)-(1.3).

Next, we give some further comments on the optimal problem (CP). As in [15], here we aim to search for a medical strategy such that the integral over the full space-time domain of the squared amount of nutrient or drug supplied (which is restricted by the control constraints) does not inflict any harm on the patient (which is expressed by the presence of the fifth summand in the cost functional $\mathcal{J}$ ). The non-negative coefficients $\beta_{Q}, \beta_{\Omega}, \alpha_{Q}, \beta_{S}, \beta_{T}, \beta_{u}$ indicate importance of conflicting targets given in the strategy, for instance, "avoid unnecessary harm to the patient" versus "quality of the approximation of $\phi_{Q}, \phi_{\Omega}$ ". In the cost functional $\mathcal{J}$, we could also add a point-wise term of the type $\int_{\Omega}\left|\sigma(\tau)-\sigma_{\Omega}\right|^{2} \mathrm{~d} x$, or we could replace the term $\int_{0}^{T} \int_{\Omega}|u|^{2} \mathrm{~d} x \mathrm{~d} t$ by a $\tau$-dependent term $\int_{0}^{\tau} \int_{\Omega}|u|^{2} \mathrm{~d} x \mathrm{~d} t$, but both would imply that we have to look for a control $u$ in a more regular space $H^{1}\left(0, T ; L^{2}(\Omega)\right)$, which is less interesting in view of practical applications (cf. [31 for further discussions on this issue). Besides, it is possible to replace $\beta_{T} \tau$ by a more general function $f(\tau)$ where $f: \mathbb{R}_{\geq 0} \rightarrow \mathbb{R}_{\geq 0}$ is continuously differentiable and increasing. In practice it would be safer for the patient (and thus more desirable) to approximate the target functions in the $L^{\infty}$ sense rather than in the $L^{2}$ sense; however, in view of the analytical difficulties that are inherent to the highly nonlinear state system (1.1)-(1.5), this presently seems to be out of reach (see also [15]). Another interesting problem would be the one including a pointwise state constraint on the variable $\phi$ of the type $\left|\phi(x, \tau)-\phi_{\Omega}(x)\right| \leq \epsilon$ for a.e. $x$, which could be reduced to an $L^{2}$-constraint $\left\|\phi(\tau)-\phi_{\Omega}\right\|_{L^{2}(\Omega)} \leq \epsilon^{\prime}$ by using possible regularity of $\phi, \phi_{\Omega}$ (if available). This leads to a more involved adjoint system and it will be the subject of future works.

Regarding the existing literature on the aspect of optimal control, we mention the works of [9, 10, 16, 17, 38 for the Cahn-Hilliard equation, [51, 63, 64] for the convective Cahn-Hilliard equation, 26, 39] for the Cahn-Hilliard-Navier-Stokes system, 54 for the Cahn-Hilliard-Darcy system, [18] for Allen-Cahn equation and [6] for a liquid crystal model. In the context of PDE constraint optimal control for diffuse interface tumor models, we have basically two recent works: [15] and [31]. In [15] the objective functional is (1.8), with the special (simpler) choices $\beta_{S}=$ $\beta_{T}=\alpha_{Q}=0$, and the state system is exactly (1.1)-(1.5) but no dependence on $\tau$ is studied. In 31 a different diffuse interface model resulting as a particular case of a more general model introduced in [32, is studied. There the distributed control appears in the $\phi$ equation, which is a Cahn-Hilliard type equation with a source of mass on the right hand side, but not depending on $\mu$. Due to the presence of the control in the Cahn-Hilliard equation, in [31] only the case of a regularized objective cost functional can be analyzed for bounded controls. Finally, we would also quote the recent paper [12, where the authors study the problem of sliding mode control for a simplified version of the model introduced in [32]. With our work we aim to provide a contribution to the theory of free terminal time optimal control in the context of diffuse interface tumor models, where the control is applied in the nutrient equation.

The rest of this paper is organized as follows. In Section 2, we formulate the general hypotheses and state some known results regarding the well-posedness, regularity as well as continuous dependence result of the state system (1.1)-(1.5). In Section 3, we study the long-time behavior of the system (1.1) - (1.5) under suitable assumption on the external source $u$, and in Section 4, we prove Lyapunov stability of local minimizers of $\mathcal{E}$ with zero mass $u=0$. The results concerning existence and first-order necessary optimality conditions for the optimal control problem (CP) 
are shown in Section 5. In Appendix, we give a brief derivation of an extended Łojasiewicz-Simon type inequality.

\section{Preliminaries}

\subsection{Notations and assumptions}

Throughout this paper, for a (real) Banach space $X$ we denote by $\|\cdot\|_{X}$ its norm, by $X^{\prime}$ its dual space, and by $\langle\cdot, \cdot\rangle_{X^{\prime}, X}$ the dual pairing between $X^{\prime}$ and $X$. If $X$ is a Hilbert space, then the inner product is denoted by $(\cdot, \cdot)_{X}$. Next, $L^{q}(\Omega), 1 \leq q \leq \infty$ denotes the usual Lebesgue space in $\Omega$ and $\|\cdot\|_{L^{q}(\Omega)}$ denotes its norm. Similarly, $W^{m, q}(\Omega), m \in \mathbb{N}, 1 \leq q \leq \infty$, denotes the usual Sobolev space with norm $\|\cdot\|_{W^{m, p}(\Omega)}$. When $q=2$, we simply indicate $W^{m, 2}(\Omega)$ by $H^{m}(\Omega)$. For simplicity, the inner product in $L^{2}(\Omega)$ will be indicated by $(\cdot, \cdot)$. Let $I$ be an interval of $\mathbb{R}^{+}$and $X$ a Banach space, the function space $L^{p}(I ; X), 1 \leq p \leq \infty$ consists of $p$-integrable functions with values in $X$. Moreover, $C_{w}(I ; X)$ denotes the topological vector space of all bounded and weakly continuous functions from $I$ to $X$, while $W^{1, p}(I, X)(1 \leq p \leq \infty)$ stands for the space of all functions $u$ such that $u, \frac{\mathrm{d} u}{\mathrm{~d} t} \in L^{p}(I ; X)$, where $\frac{\mathrm{d} u}{\mathrm{~d} t}$ denotes the vector valued distributional derivative of $u$. Bold characters will be used to denote vector spaces.

Given any function $u \in\left(H^{1}(\Omega)\right)^{\prime}$, we define the mean value by

$$
\bar{u}=|\Omega|^{-1}\langle u, 1\rangle_{\left(H^{1}\right)^{\prime}, H^{1}} .
$$

If $u \in L^{1}(\Omega)$, we simply have $\bar{u}=|\Omega|^{-1} \int_{\Omega} u \mathrm{~d} x$. We will use the notations

$$
\begin{aligned}
\dot{L}^{2}(\Omega) & =\left\{u \in L^{2}(\Omega) \mid \bar{u}=0\right\}, \\
H_{N}^{2}(\Omega) & =\left\{u \in H^{2}(\Omega) \mid \partial_{\nu} u=0 \text { a.e. on } \partial \Omega\right\}, \\
H_{N}^{4}(\Omega) & =\left\{\varphi \in H^{4}(\Omega) \mid \partial_{\nu} \varphi=\partial_{\nu} \Delta \varphi=0 \text { on } \partial \Omega\right\} .
\end{aligned}
$$

Then we have the dense and continuous embeddings $H_{N}^{2} \subset H^{1} \subset L^{2} \cong L^{2} \subset\left(H^{1}\right)^{\prime} \subset\left(H_{N}^{2}\right)^{\prime}$ (we omit to indicate the set $\Omega$ for the sake of brevity), where $\langle u, v\rangle_{\left(H^{1}\right)^{\prime}, H^{1}}=(u, v)$ and $\langle u, w\rangle_{\left(H_{N}^{2}\right)^{\prime}, H_{N}^{2}}=(u, w)$ for any $u \in L^{2}(\Omega), v \in H^{1}(\Omega)$ and $w \in H_{N}^{2}(\Omega)$. On the other hand, we observe that the operator $A:=-\Delta$ with its domain $D(A)=H_{N}^{2}(\Omega) \cap \dot{L}^{2}(\Omega)$ is a positively defined, self-adjoint operator on $D(A)$ and the spectral theorem enables us to define the powers $A^{s}$ of $A$, for $s \in \mathbb{R}$. Then the space $\left(H^{1}(\Omega)\right)^{\prime}$ can be endowed with the equivalent norm $\|u\|_{\left(H^{1}(\Omega)\right)^{\prime}}^{2}=\left\|\nabla A^{-1}(u-\bar{u})\right\|_{L^{2}(\Omega)}^{2}+|\bar{u}|^{2}$.

Throughout the paper, $C \geq 0$ will stand for a generic constant and $\mathcal{Q}(\cdot)$ for a generic positive monotone increasing function. Special dependence will be pointed out in the text if necessary.

We make the following assumptions on the nonlinear functions $P$ and $F$.

(P1) $P \in C^{2}(\mathbb{R})$ is nonnegative. There exist $\alpha_{1}>0$ and some $q \in[1,4]$ such that, for all $s \in \mathbb{R}$,

$$
\left|P^{\prime}(s)\right| \leq \alpha_{1}\left(1+|s|^{q-1}\right) .
$$

(F1) $F=F_{0}+F_{1}$, with $F_{0}, F_{1} \in C^{5}(\mathbb{R})$. There exist $\alpha_{i}>0, i=2, \ldots, 6$ and $r \in[2,6)$ such that, for all $s \in \mathbb{R}$,

$$
\begin{aligned}
& \left|F_{1}^{\prime \prime}(s)\right| \leq \alpha_{2}, \\
& \alpha_{3}\left(1+|s|^{r-2}\right) \leq F_{0}^{\prime \prime}(s) \leq \alpha_{4}\left(1+|s|^{r-2}\right), \\
& F(s) \geq \alpha_{5}|s|-\alpha_{6} .
\end{aligned}
$$


(U1) For any $T>0, u \in L^{2}\left(0, T ; L^{2}(\Omega)\right)$.

Remark 2.1. Well-posedness of problem (1.1) -1.5) with $u=0$ has been obtained in [24] under slightly weaker conditions than $(\boldsymbol{P} \mathbf{1})$ and $(\boldsymbol{F} \mathbf{1})$. On the other hand, the assumptions $(\boldsymbol{P} \mathbf{1})$, $(\boldsymbol{F} 1)$ and (U1) were indispensable for the analysis of the optimal control problem in [15]. In this paper, to avoid unnecessary technical details, we do not aim to pursue optimal assumptions on functions $F$ and $P$.

\subsection{Well-posedness and continuous dependence}

We first state the following result on well-posedness and continuous dependence of global weak solutions for problem (1.1)-(1.5) (see [24, Theorem 1, Theorem 2] for the autonomous case $u=0$ ).

Proposition 2.1. Assume that $(\boldsymbol{P} 1),(\boldsymbol{F} 1)$ and $(\mathrm{U} 1)$ are satisfied.

(1) Let $\phi_{0} \in H^{1}(\Omega)$ and $\sigma_{0} \in L^{2}(\Omega)$. Then, for every $T>0$, problem (1.1) (1.5) admits a unique weak solution on $[0, T]$ such that

$$
\begin{aligned}
& \phi \in L^{\infty}\left(0, T ; H^{1}(\Omega)\right) \cap L^{2}\left(0, T ; H_{N}^{2}(\Omega) \cap H^{3}(\Omega)\right) \cap H^{1}\left(0, T ;\left(H^{1}(\Omega)\right)^{\prime}\right), \\
& \sigma \in L^{\infty}\left(0, T ; L^{2}(\Omega)\right) \cap L^{2}\left(0, T ; H^{1}(\Omega)\right) \cap H^{1}\left(0, T ;\left(H^{1}(\Omega)\right)^{\prime}\right), \\
& \mu \in L^{2}\left(0, T ; H^{1}(\Omega)\right), \quad \sqrt{P(\phi)}(\mu-\sigma) \in L^{2}\left(0, T ; L^{2}(\Omega)\right) .
\end{aligned}
$$

In addition, the following identities are satisfied, for a.e. $t \in(0, T)$ and for any $\xi \in H^{1}(\Omega)$,

$$
\begin{aligned}
\left\langle\phi_{t}, \xi\right\rangle_{\left(H^{1}\right)^{\prime}, H^{1}}+(\nabla \mu, \nabla \xi) & =(P(\phi)(\mu-\sigma), \xi), \\
\left\langle\sigma_{t}, \xi\right\rangle_{\left(H^{1}\right)^{\prime}, H^{1}}+(\nabla \sigma, \nabla \xi) & =-(P(\phi)(\mu-\sigma), \xi)+(u, \xi),
\end{aligned}
$$

as well as $\mu=-\Delta \phi+F^{\prime}(\phi)$, a.e. in $\Omega \times(0, T)$, together with the initial conditions (1.5). Moreover, the following energy identity holds

$$
\begin{aligned}
& \mathcal{E}(\phi(t), \sigma(t))+\int_{0}^{t}\left(\|\nabla \mu\|_{L^{2}(\Omega)}^{2}+\|\nabla \sigma\|_{L^{2}(\Omega)}^{2}\right) \mathrm{d} \tau+\int_{0}^{t} \int_{\Omega} P(\phi)(\mu-\sigma)^{2} \mathrm{~d} x \mathrm{~d} \tau \\
& \quad=\mathcal{E}\left(\phi_{0}, \sigma_{0}\right)+\int_{0}^{t} \int_{\Omega} u \sigma \mathrm{d} x \mathrm{~d} \tau, \quad \forall t \in[0, T]
\end{aligned}
$$

where $\mathcal{E}$ is given by (1.6).

(2) Let $\left(\phi_{i 0}, \sigma_{i 0}\right) \in H^{1}(\Omega) \times L^{2}(\Omega)(i=1,2)$ be two initial data, $u_{i}$ be two external source terms and $\left(\phi_{i}, \sigma_{i}\right)$ be the corresponding weak solutions, $i=1,2$. Then, the following continuous dependence estimate holds

$$
\begin{gathered}
\left\|\phi_{1}-\phi_{2}\right\|_{L^{\infty}\left(0, T ;\left(H^{1}(\Omega)\right)^{\prime}\right) \cap L^{2}\left(0, T ; H^{1}(\Omega)\right)}+\left\|\sigma_{1}-\sigma_{2}\right\|_{L^{\infty}\left(0, T ;\left(H^{1}(\Omega)\right)^{\prime}\right) \cap L^{2}\left(0, T ; L^{2}(\Omega)\right)} \\
\quad \leq C_{T}\left(\left\|\phi_{10}-\phi_{20}\right\|_{\left(H^{1}(\Omega)\right)^{\prime}}+\left\|\sigma_{10}-\sigma_{20}\right\|_{\left(H^{1}(\Omega)\right)^{\prime}}+\left\|u_{1}-u_{2}\right\|_{L^{2}\left(0, T ; L^{2}(\Omega)\right)}\right)
\end{gathered}
$$

where $C_{T}>0$ is a constant depending on $\left\|\phi_{i 0}\right\|_{H^{1}(\Omega)},\left\|\sigma_{i 0}\right\|_{L^{2}(\Omega)},\left\|u_{i}\right\|_{L^{2}\left(0, T ; L^{2}(\Omega)\right)}, \Omega$ and $T$.

Remark 2.2. We note that Proposition [2.1 can be proved by means of a double approximation procedure exactly as in [24]. The presence of the external source term u only yields a minor modification in the proof, thus the details can be omitted here.

If the initial datum $\left(\phi_{0}, \sigma_{0}\right)$ is smoother, the following result on well-posedness and continuous dependence of global strong solutions was obtained in [15, Theorem 2.1, Theorem 2.2]. 
Proposition 2.2. Assume that (P1), (F1) and (U1) are satisfied. Let $\phi_{0} \in H_{N}^{2}(\Omega) \cap H^{3}(\Omega)$ and $\sigma_{0} \in H^{1}(\Omega)$.

(1) For every $T>0$, problem (1.1) -(1.5) admits a unique strong solution on $[0, T]$ such that

$$
\begin{aligned}
& \phi \in L^{\infty}\left(0, T ; H_{N}^{2}(\Omega) \cap H^{3}(\Omega)\right) \cap L^{2}\left(0, T ; H_{N}^{4}(\Omega)\right) \cap H^{1}\left(0, T ; H^{1}(\Omega)\right), \\
& \mu \in L^{\infty}\left(0, T ; H^{1}(\Omega)\right) \cap L^{2}\left(0, T ; H_{N}^{2}(\Omega)\right), \\
& \sigma \in C\left([0, T] ; H^{1}(\Omega)\right) \cap L^{2}\left(0, T ; H_{N}^{2}(\Omega)\right) \cap H^{1}\left(0, T ; L^{2}(\Omega)\right) .
\end{aligned}
$$

(2) There exists a constant $K_{1}>0$, depending on $\left\|\phi_{0}\right\|_{H^{3}(\Omega)},\left\|\sigma_{0}\right\|_{H^{1}(\Omega)},\|u\|_{L^{2}\left(0, T ; L^{2}(\Omega)\right)}, \Omega$ and $T$ such that the strong solution $(\phi, \mu, \sigma)$ satisfies

$$
\begin{aligned}
& \|\phi\|_{L^{\infty}\left(0, T ; H^{3}(\Omega)\right) \cap L^{2}\left(0, T ; H^{4}(\Omega)\right) \cap H^{1}\left(0, T ; H^{1}(\Omega)\right)}+\|\mu\|_{L^{\infty}\left(0, T ; H^{1}(\Omega)\right) \cap L^{2}\left(0, T ; H^{2}(\Omega)\right)} \\
& \quad+\|\sigma\|_{C\left([0, T] ; H^{1}(\Omega)\right) \cap L^{2}\left(0, T ; H_{N}^{2}(\Omega)\right) \cap H^{1}\left(0, T ; L^{2}(\Omega)\right)} \leq K_{1} .
\end{aligned}
$$

(3) For arbitrary $u_{i} \in L^{2}\left(0, T ; L^{2}(\Omega)\right)(i=1,2), \phi_{0} \in H_{N}^{2}(\Omega) \cap H^{3}(\Omega)$ and $\sigma_{0} \in H^{1}(\Omega)$, let $\left(\phi_{i}, \sigma_{i}\right)$ be the corresponding strong solutions. Then there exists a constant $K_{2}>0$, depending on $\left\|u_{i}\right\|_{L^{2}\left(0, T ; L^{2}\right)}, \Omega, T,\left\|\phi_{0}\right\|_{H^{3}}$ and $\left\|\sigma_{0}\right\|_{H^{1}}$, such that

$$
\begin{aligned}
& \left\|\phi_{1}-\phi_{2}\right\|_{L^{\infty}\left(0, T ; H^{1}(\Omega)\right) \cap L^{2}\left(0, T ; H^{3}(\Omega)\right) \cap H^{1}\left(0, T ;\left(H^{1}(\Omega)\right)^{\prime}\right)}+\left\|\mu_{1}-\mu_{2}\right\|_{L^{2}\left(0, T ; H^{1}(\Omega)\right)} \\
& \quad+\left\|\sigma_{1}-\sigma_{2}\right\|_{C\left([0, T] ; H^{1}(\Omega)\right) \cap L^{2}\left(0, T ; H^{2}(\Omega)\right) \cap H^{1}\left(0, T ; L^{2}(\Omega)\right)} \leq K_{2}\left\|u_{1}-u_{2}\right\|_{L^{2}\left(0, T ; L^{2}(\Omega)\right)} .
\end{aligned}
$$

\section{Long-time Dynamics}

In order to study the long-time behavior of problem (1.1)-(1.5), we make the following additional assumptions.

(P2) $P(s)>0$, for all $s \in \mathbb{R}$.

(F2) $F(s)$ is real analytic, for all $s \in \mathbb{R}$.

(U2) $u \in L^{1}\left(0,+\infty ; L^{2}(\Omega)\right) \cap L^{2}\left(0,+\infty ; L^{2}(\Omega)\right)$ and satisfies the decay condition

$$
\sup _{t \geq 0}(1+t)^{3+\rho}\|u(t)\|_{L^{2}(\Omega)}<+\infty, \quad \text { for some } \rho>0 .
$$

The main result of this section reads as follows.

Theorem 3.1 (Convergence to equilibrium). Assume that (P1), (P2), (F1), (F2) and (U2) are satisfied. For any $\phi_{0} \in H^{1}(\Omega)$ and $\sigma_{0} \in L^{2}(\Omega)$, problem (1.1)-(1.5) admits a unique global weak solution $(\phi, \mu, \sigma)$ such that

$$
\lim _{t \rightarrow+\infty}\left(\left\|\phi(t)-\phi_{\infty}\right\|_{H^{2}(\Omega)}+\left\|\sigma(t)-\sigma_{\infty}\right\|_{L^{2}(\Omega)}+\left\|\mu(t)-\mu_{\infty}\right\|_{L^{2}(\Omega)}\right)=0,
$$

where $\left(\phi_{\infty}, \mu_{\infty}, \sigma_{\infty}\right)$ satisfies

$$
\begin{cases}-\Delta \phi_{\infty}+F^{\prime}\left(\phi_{\infty}\right)=\mu_{\infty}, & \text { in } \Omega, \\ \partial_{\nu} \phi_{\infty}=0, & \text { on } \partial \Omega, \\ \int_{\Omega}\left(\phi_{\infty}+\sigma_{\infty}\right) \mathrm{d} x=\int_{\Omega}\left(\phi_{0}+\sigma_{0}\right) \mathrm{d} x+\int_{0}^{+\infty} \int_{\Omega} u \mathrm{~d} x \mathrm{~d} t,\end{cases}
$$

with $\mu_{\infty}$ and $\sigma_{\infty}$ being two constants given by

$$
\sigma_{\infty}=\mu_{\infty}
$$




$$
\mu_{\infty}=|\Omega|^{-1} \int_{\Omega} F^{\prime}\left(\phi_{\infty}\right) d x
$$

Moreover, the following estimates on convergence rate hold

$$
\begin{aligned}
& \left\|\phi(t)-\phi_{\infty}\right\|_{H^{1}(\Omega)}+\left\|\sigma(t)-\sigma_{\infty}\right\|_{L^{2}(\Omega)} \leq C(1+t)^{-\min \left\{\frac{\theta}{1-2 \theta}, \frac{\rho}{2}\right\}}, \quad \forall t \geq 0, \\
& \left\|\mu(t)-\mu_{\infty}\right\|_{L^{2}(\Omega)} \leq C(1+t)^{-\frac{1}{2} \min \left\{\frac{\theta}{1-2 \theta}, \frac{\rho}{2}\right\}}, \quad \forall t \geq 0,
\end{aligned}
$$

where $C>0$ is a constant depending on $\left\|\phi_{0}\right\|_{H^{1}(\Omega)},\left\|\sigma_{0}\right\|_{L^{2}(\Omega)},\left\|\phi_{\infty}\right\|_{H^{1}(\Omega)},\|u\|_{L^{1}\left(0,+\infty ; L^{2}(\Omega)\right)}$, $\|u\|_{L^{2}\left(0,+\infty ; L^{2}(\Omega)\right)}$ and $\Omega ; \theta \in\left(0, \frac{1}{2}\right)$ is a constant depending on $\phi_{\infty}$.

The proof of Theorem 3.1 consists of several steps. We first derive some uniform-in-time a priori estimates on the solution $(\phi, \mu, \sigma)$. Then we give a characterization on the $\omega$-limit set. Finally, we prove the convergence of the trajectories and polynomial decay by means of a proper Łojasiewicz-Simon inequality (see Lemma 3.1).

\subsection{Uniform-in-time estimates}

We proceed to derive uniform-in-time estimates for the global weak solution to problem (1.1)(1.5) under the additional assumption (U2) for $u$.

First estimate. Adding (2.5) with (2.6), testing the resultant by $\xi=1$, after integration by parts, and then integrating with respect to time, we have

$$
\int_{\Omega}(\phi(t)+\sigma(t)) \mathrm{d} x=\int_{\Omega}\left(\phi_{0}+\sigma_{0}\right) \mathrm{d} x+\int_{0}^{t} \int_{\Omega} u \mathrm{~d} x \mathrm{~d} \tau, \quad \forall t \geq 0,
$$

which together with (U2) implies the bound of total mass:

$$
\left|\int_{\Omega}(\phi(t)+\sigma(t)) \mathrm{d} x\right| \leq\left|\int_{\Omega}\left(\phi_{0}+\sigma_{0}\right) \mathrm{d} x\right|+\|u\|_{L^{1}\left(0,+\infty ; L^{1}(\Omega)\right)}, \quad \forall t \geq 0 .
$$

Second estimate. By the Hölder inequality and the Cauchy-Schwarz inequality, we infer that

$$
\left|\int_{\Omega} u \sigma \mathrm{d} x\right| \leq\|u\|_{L^{2}(\Omega)}\|\sigma\|_{L^{2}(\Omega)} \leq \frac{1}{2}\|u\|_{L^{2}(\Omega)}\|\sigma\|_{L^{2}(\Omega)}^{2}+\frac{1}{2}\|u\|_{L^{2}(\Omega)} .
$$

From the energy identity (2.7), we have

$$
\begin{aligned}
& \mathcal{E}(\phi(t), \sigma(t))+\int_{0}^{t}\left(\|\nabla \mu\|_{L^{2}(\Omega)}^{2}+\|\nabla \sigma\|_{L^{2}(\Omega)}^{2}\right) \mathrm{d} \tau+\int_{0}^{t} \int_{\Omega} P(\phi)(\mu-\sigma)^{2} \mathrm{~d} x \mathrm{~d} \tau \\
& \quad \leq \mathcal{E}\left(\phi_{0}, \sigma_{0}\right)+\frac{1}{2} \int_{0}^{t}\|u\|_{L^{2}}\left(\|\sigma\|_{L^{2}}^{2}+1\right) \mathrm{d} t, \quad \forall t \geq 0 .
\end{aligned}
$$

Denote

$$
\widehat{\mathcal{E}}(t)=\widehat{\mathcal{E}}(\phi(t), \sigma(t))+\max \left\{\alpha_{6}, 1\right\},
$$

where the constant $\alpha_{6}$ is given in (F1). From the classical Bellman-Gronwall inequality (cf. [3]), it follows that

$$
\begin{aligned}
\widehat{\mathcal{E}}(t) & \leq \widehat{\mathcal{E}}(0) e^{\int_{0}^{t}\|u(\tau)\|_{L^{2}} \mathrm{~d} \tau} \\
& \leq\left(\mathcal{E}\left(\phi_{0}, \sigma_{0}\right)+\max \left\{\alpha_{6}, 1\right\}\right) e^{\|u\|_{L^{1}\left(0,+\infty ; L^{2}(\Omega)\right)}}, \quad \forall t \geq 0,
\end{aligned}
$$


which further implies

$$
\begin{aligned}
& \sup _{t \geq 0}\left(\|\phi(t)\|_{H^{1}(\Omega)}+\|\sigma(t)\|_{L^{2}(\Omega)}+\|F(\phi(t))\|_{L^{1}(\Omega)}\right) \leq C, \\
& \int_{0}^{+\infty}\left(\|\nabla \mu\|_{L^{2}(\Omega)}^{2}+\|\nabla \sigma\|_{L^{2}(\Omega)}^{2}+\int_{\Omega} P(\phi)(\mu-\sigma)^{2} \mathrm{~d} x\right) \mathrm{d} t \leq C,
\end{aligned}
$$

where $C$ is a positive constant depending on $\left\|\phi_{0}\right\|_{H^{1}(\Omega)},\left\|\sigma_{0}\right\|_{L^{2}(\Omega)},\|u\|_{L^{1}\left(0,+\infty ; L^{2}(\Omega)\right)}, \Omega$ and $\alpha_{6}$.

Third estimate. Higher-order estimates will be carried out in a formal way, which can be justified rigorously by means of a suitable approximation procedure (cf. [24, Section 3]). Testing (2.5) by $\xi=\mu_{t}$ and (2.6) by $\xi=\sigma_{t}$, adding the resultants together, we obtain (see [24, (4.3)]):

$$
\begin{aligned}
& \frac{1}{2} \frac{\mathrm{d}}{\mathrm{d} t}\left(\|\nabla \mu\|_{L^{2}(\Omega)}^{2}+\|\nabla \sigma\|_{L^{2}(\Omega)}^{2}+\int_{\Omega} P(\phi)(\mu-\sigma)^{2} \mathrm{~d} x\right)+\left\|\nabla \phi_{t}\right\|_{L^{2}(\Omega)}^{2}+\left\|\sigma_{t}\right\|_{L^{2}(\Omega)}^{2} \\
& \quad=\frac{1}{2} \int_{\Omega} P^{\prime}(\phi) \phi_{t}(\mu-\sigma)^{2} \mathrm{~d} x-\int_{\Omega} F^{\prime \prime}(\phi) \phi_{t}^{2} \mathrm{~d} x+\int_{\Omega} u \sigma_{t} \mathrm{~d} x . \\
& \quad:=I_{1}+I_{2}+I_{3} .
\end{aligned}
$$

As in [24, we infer from the Hölder inequality that

$$
\begin{aligned}
I_{1} & \leq \frac{1}{2}\left\|P^{\prime}(\phi)\right\|_{L^{2}(\Omega)}\left\|\phi_{t}\right\|_{L^{6}(\Omega)}\|\mu-\sigma\|_{L^{6}(\Omega)}^{2} \\
& \leq C\left\|P^{\prime}(\phi)\right\|_{L^{2}(\Omega)}\left\|\phi_{t}\right\|_{H^{1}(\Omega)}\|\mu-\sigma\|_{H^{1}(\Omega)}^{2} .
\end{aligned}
$$

Next, by the Poincaré-Wirtinger inequality, and using (1.1), we see that

$$
\begin{aligned}
\left\|\phi_{t}\right\|_{H^{1}(\Omega)} & \leq\left(1+c_{\Omega}\right)\left\|\nabla \phi_{t}\right\|_{L^{2}(\Omega)}+|\Omega|^{\frac{1}{2}}\left|\overline{\phi_{t}}\right| \\
& \leq\left(1+c_{\Omega}\right)\left\|\nabla \phi_{t}\right\|_{L^{2}(\Omega)}+|\Omega|^{-\frac{1}{2}}\left|\int_{\Omega} P(\phi)(\mu-\sigma) \mathrm{d} x\right| \\
& \leq\left(1+c_{\Omega}\right)\left\|\nabla \phi_{t}\right\|_{L^{2}(\Omega)}+|\Omega|^{-\frac{1}{2}}\|\sqrt{P(\phi)}\|_{L^{2}(\Omega)}\|\sqrt{P(\phi)}(\mu-\sigma)\|_{L^{2}(\Omega)} .
\end{aligned}
$$

Besides, from the Sobolev embedding theorem, (1.2), (3.13) and (F1) it follows

$$
\begin{aligned}
|\bar{\mu}| & =|\Omega|^{-1}\left|\int_{\Omega}\left(-\Delta \phi+F^{\prime}(\phi)\right) \mathrm{d} x\right| \\
& =|\Omega|^{-1}\left|\int_{\Omega} F^{\prime}(\phi) d x\right| \\
& \leq C\left(1+\|\phi\|_{L^{r-1}(\Omega)}^{r-1}\right) \\
& \leq C\left(1+\|\phi\|_{H^{1}(\Omega)}^{r-1}\right) \leq C .
\end{aligned}
$$

Thus, by the Poincaré-Wirtinger inequality again, we have

$$
\|\mu\|_{H^{1}(\Omega)} \leq\left(1+c_{\Omega}\right)\|\nabla \mu\|_{L^{2}(\Omega)}+|\Omega|^{\frac{1}{2}}|\bar{\mu}| \leq C\left(\|\nabla \mu\|_{L^{2}(\Omega)}+1\right) .
$$

Besides, we also notice that

$$
\|\sigma\|_{H^{1}(\Omega)} \leq\|\nabla \sigma\|_{L^{2}(\Omega)}+\|\sigma\|_{L^{2}(\Omega)} \leq\|\nabla \sigma\|_{L^{2}(\Omega)}+C .
$$

Using the above estimates, (P1) and (3.13), we deduce that

$$
I_{1} \leq C\left\|P^{\prime}(\phi)\right\|_{L^{2}(\Omega)}\left[\left(1+c_{\Omega}\right)\left\|\nabla \phi_{t}\right\|_{L^{2}(\Omega)}+|\Omega|^{-\frac{1}{2}}\|\sqrt{P(\phi)}\|_{L^{2}(\Omega)}\|\sqrt{P(\phi)}(\mu-\sigma)\|_{L^{2}(\Omega)}\right]
$$




$$
\begin{aligned}
& \times\left(\|\mu\|_{H^{1}(\Omega)}^{2}+\|\sigma\|_{H^{1}(\Omega)}^{2}\right) \\
\leq & C\left(\left\|\nabla \phi_{t}\right\|_{L^{2}(\Omega)}+\|\sqrt{P(\phi)}(\mu-\sigma)\|_{L^{2}(\Omega)}\right)\left(\|\nabla \mu\|_{L^{2}(\Omega)}^{2}+\|\nabla \sigma\|_{L^{2}(\Omega)}^{2}+1\right) \\
\leq & \frac{1}{4}\left\|\nabla \phi_{t}\right\|_{L^{2}(\Omega)}^{2}+C\left(\|\nabla \mu\|_{L^{2}(\Omega)}^{4}+\|\nabla \sigma\|_{L^{2}(\Omega)}^{4}+\|\sqrt{P(\phi)}(\mu-\sigma)\|_{L^{2}(\Omega)}^{2}+1\right) .
\end{aligned}
$$

Next, using the fact $\int_{\Omega} \Delta \mu \mathrm{d} x=0$ (cf. (1.4) ), we have

$$
\|\Delta \mu\|_{\left(H^{1}(\Omega)\right)^{\prime}}=\|\nabla \mu\|_{L^{2}(\Omega)} .
$$

It follows from equation (1.1) that

$$
\left\|-\phi_{t}+P(\phi)(\mu-\sigma)\right\|_{\left(H^{1}(\Omega)\right)^{\prime}}=\|\nabla \mu\|_{L^{2}(\Omega)} .
$$

Then on account of (P1) and (3.13), we obtain

$$
\begin{aligned}
\left\|\phi_{t}\right\|_{\left(H^{1}(\Omega)\right)^{\prime}} & \leq\left\|-\phi_{t}+P(\phi)(\mu-\sigma)\right\|_{\left(H^{1}(\Omega)\right)^{\prime}}+\|P(\phi)(\mu-\sigma)\|_{\left(H^{1}(\Omega)\right)^{\prime}} \\
& \leq\|\nabla \mu\|_{L^{2}(\Omega)}+\|P(\phi)(\mu-\sigma)\|_{L^{\frac{6}{5}}(\Omega)} \\
& \leq\|\nabla \mu\|_{L^{2}(\Omega)}+\|\sqrt{P(\phi)}\|_{L^{3}(\Omega)}\|\sqrt{P(\phi)}(\mu-\sigma)\|_{L^{2}(\Omega)} \\
& \leq\|\nabla \mu\|_{L^{2}(\Omega)}+C\left(1+\|\phi\|_{L^{\frac{q}{2}}(\Omega)}^{\frac{3 q}{2}}\right)\|\sqrt{P(\phi)}(\mu-\sigma)\|_{L^{2}(\Omega)} \\
& \leq\|\nabla \mu\|_{L^{2}(\Omega)}+C\|\sqrt{P(\phi)}(\mu-\sigma)\|_{L^{2}(\Omega)} .
\end{aligned}
$$

Collecting (3.16) and (3.20), we get

$$
\begin{aligned}
I_{2} & \leq \alpha_{2}\left\|\phi_{t}\right\|_{L^{2}(\Omega)}^{2} \\
& \leq C\left\|\phi_{t}\right\|_{H^{1}(\Omega)}\left\|\phi_{t}\right\|_{\left(H^{1}(\Omega)\right)^{\prime}} \\
& \leq C\left(\left\|\nabla \phi_{t}\right\|_{L^{2}(\Omega)}+\|\sqrt{P(\phi)}(\mu-\sigma)\|_{L^{2}(\Omega)}\right)\left(\|\nabla \mu\|_{L^{2}(\Omega)}+C\|\sqrt{P(\phi)}(\mu-\sigma)\|_{L^{2}(\Omega)}\right) \\
& \leq \frac{1}{4}\left\|\nabla \phi_{t}\right\|_{L^{2}(\Omega)}^{2}+C\|\nabla \mu\|_{L^{2}(\Omega)}^{2}+\|\sqrt{P(\phi)}(\mu-\sigma)\|_{L^{2}(\Omega)}^{2} .
\end{aligned}
$$

Concerning $I_{3}$, it holds that

$$
I_{3} \leq\|u\|_{L^{2}(\Omega)}\left\|\sigma_{t}\right\|_{L^{2}(\Omega)} \leq \frac{1}{2}\left\|\sigma_{t}\right\|_{L^{2}(\Omega)}^{2}+\frac{1}{2}\|u\|_{L^{2}(\Omega)}^{2} .
$$

Set

$$
\mathcal{A}(t):=\|\nabla \mu(t)\|_{L^{2}(\Omega)}^{2}+\|\nabla \sigma(t)\|_{L^{2}(\Omega)}^{2}+\|\sqrt{P(\phi(t))}(\mu(t)-\sigma(t))\|_{L^{2}(\Omega)}^{2} .
$$

The estimate (3.14) implies that

$$
\int_{0}^{+\infty} \mathcal{A}(t) d t<+\infty
$$

From (3.15) and the above estimates for reminder terms $I_{1}, I_{2}, I_{3}$, we deduce that

$$
\frac{\mathrm{d}}{\mathrm{d} t} \mathcal{A}(t)+\left\|\nabla \phi_{t}\right\|_{L^{2}(\Omega)}^{2}+\left\|\sigma_{t}\right\|_{L^{2}(\Omega)}^{2} \leq C \mathcal{A}(t)^{2}+\|u\|_{L^{2}(\Omega)}^{2}+C,
$$

where $C$ is a positive constant depending on $\left\|\phi_{0}\right\|_{H^{1}(\Omega)},\left\|\sigma_{0}\right\|_{L^{2}(\Omega)},\|u\|_{L^{1}\left(0,+\infty ; L^{2}(\Omega)\right)}, \Omega, \alpha_{1}, \alpha_{2}$, $\alpha_{4}$ and $\alpha_{6}$. Using (3.22) and the assumption $u \in L^{2}\left(0,+\infty ; L^{2}(\Omega)\right)$, then from [65, Lemma 6.2.1] we obtain, for any $\delta \in(0,1)$,

$$
\mathcal{A}(t+\delta) \leq C\left(1+\delta^{-1}\right), \quad \forall t \geq 0,
$$


where $C$ is a positive constant depending on the initial data $\left\|\phi_{0}\right\|_{H^{1}(\Omega)},\left\|\sigma_{0}\right\|_{L^{2}(\Omega)}, \Omega, \alpha_{1}, \alpha_{2}, \alpha_{4}$, $\alpha_{6},\|u\|_{L^{1}\left(0,+\infty ; L^{2}(\Omega)\right)}$ and $\|u\|_{L^{2}\left(0,+\infty ; L^{2}(\Omega)\right)}$. Besides, by [65, Lemma 6.2.1], we can also conclude that

$$
\lim _{t \rightarrow+\infty} \mathcal{A}(t)=0
$$

Fourth estimate. Combining the estimates (3.17)-(3.19) and (3.24), we have

$$
\begin{aligned}
\|\mu(t)\|_{H^{1}(\Omega)} \leq C, & \forall t \geq \delta, \\
\|\sigma(t)\|_{H^{1}(\Omega)} \leq C, & \forall t \geq \delta .
\end{aligned}
$$

Testing (1.2) by $-\Delta \phi$, using (F1), (3.13) and (3.26), we see that

$$
\begin{aligned}
\|\Delta \phi\|_{L^{2}(\Omega)}^{2} & =-\int_{\Omega} \Delta \phi \mu \mathrm{d} x+\int_{\Omega} F^{\prime}(\phi) \Delta \phi \mathrm{d} x \\
& \leq \frac{1}{2}\|\mu\|_{L^{2}(\Omega)}^{2}+\frac{1}{2}\|\Delta \phi\|_{L^{2}(\Omega)}^{2}-\int_{\Omega} F^{\prime \prime}(\phi)|\nabla \phi|^{2} \mathrm{~d} x \\
& \leq \frac{1}{2}\|\mu\|_{L^{2}(\Omega)}^{2}+\frac{1}{2}\|\Delta \phi\|_{L^{2}(\Omega)}^{2}+\alpha_{2}\|\nabla \phi\|_{L^{2}(\Omega)}^{2} \\
& \leq \frac{1}{2}\|\Delta \phi\|_{L^{2}(\Omega)}^{2}+C .
\end{aligned}
$$

Then, by means of elliptic estimates, we obtain

$$
\|\phi(t)\|_{H^{2}(\Omega)} \leq C\left(\|\Delta \phi(t)\|_{L^{2}(\Omega)}+\|\phi(t)\|_{L^{2}(\Omega)}\right) \leq C, \quad \forall t \geq \delta .
$$

The above estimate combined with (F1), (3.13), (3.26) and the Sobolev embedding theorem $H^{2} \hookrightarrow L^{\infty}$ further yields that

$$
\begin{aligned}
\|\phi(t)\|_{H^{3}(\Omega)} & \leq C\left(\|\mu(t)\|_{H^{1}(\Omega)}+\left\|F^{\prime}(\phi(t))\right\|_{H^{1}(\Omega)}+\|\phi(t)\|_{L^{2}(\Omega)}\right) \\
& \leq C+C\left(1+\|\phi(t)\|_{L^{\infty}(\Omega)}^{r-1}\right)+C\left(1+\|\phi(t)\|_{L^{\infty}(\Omega)}^{r-2}\right)\|\nabla \phi(t)\|_{L^{2}(\Omega)} \\
& \leq C, \quad \forall t \geq \delta .
\end{aligned}
$$

Fifth estimate. We deduce from (U2), (3.14), (3.16), (3.24) and the inequality (3.23) that

$$
\int_{t}^{t+1}\left(\left\|\phi_{t}(\tau)\right\|_{H^{1}(\Omega)}^{2}+\left\|\sigma_{t}(\tau)\right\|_{L^{2}(\Omega)}^{2}\right) \mathrm{d} \tau \leq C, \quad \forall t \geq \delta .
$$

On the other hand, since

$$
\|P(\phi)(\sigma-\mu)\|_{L^{2}(\Omega)} \leq\|P(\phi)\|_{L^{\infty}(\Omega)}\left(\|\sigma\|_{L^{2}(\Omega)}+\|\mu\|_{L^{2}(\Omega)}\right),
$$

and

$$
\begin{aligned}
\|\nabla(P(\phi)(\sigma-\mu))\|_{L^{2}(\Omega)} \leq & \left\|P^{\prime}(\phi)\right\|_{L^{\infty}(\Omega)}\|\nabla \phi\|_{L^{\infty}(\Omega)}\left(\|\sigma\|_{L^{2}(\Omega)}+\|\mu\|_{L^{2}(\Omega)}\right) \\
& +\|P(\phi)\|_{L^{\infty}(\Omega)}\left(\|\nabla \mu\|_{L^{2}(\Omega)}+\|\nabla \sigma\|_{L^{2}(\Omega)}\right),
\end{aligned}
$$

it follows from (3.26) $-(3.28)$ that

$$
\int_{t}^{t+1}\|P(\phi(\tau))(\sigma(\tau)-\mu(\tau))\|_{H^{1}(\Omega)}^{2} \mathrm{~d} \tau \leq C, \quad \forall t \geq \delta .
$$


Then, by comparison in the equations (1.1) and (1.3), we infer from (3.29), (3.30), (U2) and the elliptic regularity theory that

$$
\int_{t}^{t+1}\left(\|\mu(\tau)\|_{H^{3}(\Omega)}^{2}+\|\sigma(\tau)\|_{H^{2}(\Omega)}^{2}\right) \mathrm{d} \tau \leq C, \quad \forall t \geq \delta .
$$

Applying the elliptic regularity theory to (1.2) together with the above estimate and (3.28), we finally arrive at

$$
\int_{t}^{t+1}\|\phi(\tau)\|_{H^{5}(\Omega)}^{2} \mathrm{~d} \tau \leq C, \quad \forall t \geq \delta .
$$

Remark 3.1. (1) Since $\delta \in(0,1)$ can be taken arbitrary small, the estimate (3.24) actually implies that the global weak solution $(\phi, \sigma)$ becomes a strong one once $t>0$.

(2) If we assume in addition $\phi_{0} \in H_{N}^{2}(\Omega) \cap H^{3}(\Omega), \sigma_{0} \in H^{1}(\Omega)$, then it easily follows that

$$
\mathcal{A}(t) \leq C, \quad \forall t \geq 0
$$

where $C$ is a positive constant depending on the initial data $\left\|\phi_{0}\right\|_{H^{3}(\Omega)},\left\|\sigma_{0}\right\|_{H^{1}(\Omega)}, \Omega, \alpha_{1}, \alpha_{2}$, $\alpha_{4}, \alpha_{6},\|u\|_{L^{1}\left(0,+\infty ; L^{2}(\Omega)\right)}$ and $\|u\|_{L^{2}\left(0,+\infty ; L^{2}(\Omega)\right)}$. In a similar fashion as above, we see that the previous higher-order estimates hold for all $t \geq 0$.

(3) It is worth mentioning that our higher-order estimates improve the estimate (2.8) that was obtained in [15], under the additional assumption (U2).

\subsection{Proof of Theorem 3.1; convergence to a single equilibrium}

For any initial datum $\left(\phi_{0}, \sigma_{0}\right) \in H^{1}(\Omega) \times L^{2}(\Omega)$, we define the corresponding $\omega$-limit set

$$
\begin{gathered}
\omega\left(\phi_{0}, \sigma_{0}\right)=\left\{\left(\phi_{\infty}, \sigma_{\infty}\right) \in\left(H_{N}^{2}(\Omega) \cap H^{3}(\Omega)\right) \times H^{1}(\Omega): \exists\left\{t_{n}\right\} \nearrow+\infty\right. \text { such that } \\
\left.\left(\phi\left(t_{n}\right), \sigma\left(t_{n}\right)\right) \rightarrow\left(\phi_{\infty}, \sigma_{\infty}\right) \text { in } H^{2}(\Omega) \times L^{2}(\Omega)\right\} .
\end{gathered}
$$

From the uniform-in-time estimates (3.14), (3.26) -(3.28), and keeping the mass constraint (3.8) in mind, one can easily adapt the argument in [11, Section 6] to deduce the following characterization on $\omega\left(\phi_{0}, \sigma_{0}\right)$.

Proposition 3.1. Assume that (P1), (F1), (U2) are satisfied. For any initial datum $\left(\phi_{0}, \sigma_{0}\right) \in$ $H^{1}(\Omega) \times L^{2}(\Omega)$, the associated $\omega$-limit set $\omega\left(\phi_{0}, \sigma_{0}\right)$ is non-empty. For any element $\left(\phi_{\infty}, \sigma_{\infty}\right) \in$ $\omega\left(\phi_{0}, \sigma_{0}\right), \sigma_{\infty}$ is a constant and $\left(\phi_{\infty}, \sigma_{\infty}\right)$ satisfies the stationary problem (3.3). Besides, $\mu_{\infty}$ is a constant given by (3.5) and the following relation holds

$$
P\left(\phi_{\infty}\right)\left(\sigma_{\infty}-\mu_{\infty}\right)=0, \text { a.e. in } \Omega \text {. }
$$

In particular, the relation (3.34) and the positivity assumption on $P(\cdot)$ (recall (P2)) immediately yield the following property.

Corollary 3.1. Under the assumptions of Proposition 3.1, if, in addition, (P2) is fulfilled, then $\left(\phi_{\infty}, \mu_{\infty}, \sigma_{\infty}\right)$ satisfies the elliptic problem (3.3) with relations (3.4) and (3.5).

Next, given any initial datum $\left(\phi_{0}, \sigma_{0}\right) \in H^{1}(\Omega) \times L^{2}(\Omega)$ and source term $u$ satisfying (U2), we denote by

$$
m_{\infty}:=|\Omega|^{-1}\left(\int_{\Omega}\left(\phi_{0}+\sigma_{0}\right) \mathrm{d} x+\int_{0}^{+\infty} \int_{\Omega} u \mathrm{~d} x \mathrm{~d} t\right)
$$

the total mass at infinity time (cf. (3.8)). Then we are able to derive the following gradient inequality of Łojasiewicz-Simon type, which plays a crucial role in the study of long-time behavior of problem (1.1)-(1.5). 
Lemma 3.1 (Łojasiewicz-Simon Inequality). Let (F1), (F2), (P1), (P2) and (U2) be satisfied. Suppose that $\left(\phi_{\infty}, \mu_{\infty}, \sigma_{\infty}\right)$ is a solution to the elliptic problem (3.3) together with (3.4), (3.5) and $m_{\infty}$ is a constant given by (3.35). Then there exist constants $\theta \in\left(0, \frac{1}{2}\right)$ and $\beta>0$, depending on $\phi_{\infty}, m_{\infty}$ and $\Omega$, such that for any $(\phi, \sigma) \in H_{N}^{2}(\Omega) \times H^{1}(\Omega)$ satisfying

$$
\begin{aligned}
& \left\|\phi-\phi_{\infty}\right\|_{H^{1}(\Omega)}<\beta, \\
& \int_{\Omega}(\phi+\sigma) \mathrm{d} x+m_{u}|\Omega|=\int_{\Omega}\left(\phi_{\infty}+\sigma_{\infty}\right) \mathrm{d} x=m_{\infty}|\Omega|,
\end{aligned}
$$

where $m_{u}$ is a certain constant fulfiling $\left|m_{u}\right| \leq|\Omega|^{-\frac{1}{2}}\|u\|_{L^{1}\left(0,+\infty ; L^{2}(\Omega)\right)}$, then we have

$$
\begin{aligned}
& \|\mu-\bar{\mu}\|_{\left(H^{1}(\Omega)\right)^{\prime}}+C\|\nabla \sigma\|_{L^{2}(\Omega)}+C\|\sqrt{P(\phi)}(\mu-\sigma)\|_{L^{2}(\Omega)}+C\left|m_{u}\right|^{\frac{1}{2}} \\
& \quad \geq\left|\mathcal{E}(\phi, \sigma)-\mathcal{E}\left(\phi_{\infty}, \sigma_{\infty}\right)\right|^{1-\theta} .
\end{aligned}
$$

Here, $\mu=-\Delta \phi+F^{\prime}(\phi)$ and $C>0$ is a constant depending on $\Omega, \phi_{\infty}, m_{\infty},\|\phi\|_{H^{2}(\Omega)}$, $\|\sigma\|_{H^{1}(\Omega)}$ and $\|u\|_{L^{1}\left(0,+\infty ; L^{2}(\Omega)\right)}$.

Remark 3.2. The proof of Lemma 3.1 will be postponed to the Appendix. The constant $m_{u}$ in (3.37) can be viewed as a control on the deviation of $|\Omega|^{-1} \int_{\Omega}(\phi+\sigma) \mathrm{d} x$ from the mean value of the total mass at infinity time $m_{\infty}$ (see (3.46) below for its precise definition, when it is connected with the evolution problem (1.1)-(1.5) ).

After the previous preparations, we are in a position to complete the proof of Theorem 3.1 .

Step 1. Convergence of the free energy $\mathcal{E}(\phi(t), \sigma(t))$. Let $(\phi(t), \sigma(t))$ be the global weak solution to problem (1.1)-(1.5) subject to the initial datum $\left(\phi_{0}, \sigma_{0}\right) \in H^{1}(\Omega) \times L^{2}(\Omega)$ and the source term $u$ satisfying (U2). Thanks to Remark 3.1, the global weak solution $(\phi(t), \sigma(t))$ becomes a strong one as $t \geq \delta>0$ and is uniformly bounded in $H^{3}(\Omega) \times H^{1}(\Omega)$ for all $t \geq \delta$. Then by the energy identity (1.7), Young's inequality and (3.13), we are able to obtain the following energy inequality, for a.e. $t \geq \delta$,

$$
\frac{\mathrm{d}}{\mathrm{d} t} \mathcal{E}(\phi, \sigma)+\|\nabla \mu\|_{L^{2}(\Omega)}^{2}+\|\nabla \sigma\|_{L^{2}(\Omega)}^{2}+\int_{\Omega} P(\phi)(\mu-\sigma)^{2} \mathrm{~d} x \leq K\|u\|_{L^{2}(\Omega)},
$$

where $K>0$ is a constant depending on $\left\|\phi_{0}\right\|_{H^{1}(\Omega)},\left\|\sigma_{0}\right\|_{L^{2}(\Omega)},\|u\|_{L^{1}\left(0,+\infty ; L^{2}(\Omega)\right)}, \Omega$ and $\alpha_{6}$. Denote

$$
y(t)=\int_{t}^{+\infty}\|u(\tau)\|_{L^{2}(\Omega)} \mathrm{d} \tau
$$

By assumption (U2), there exist some positive constants $C_{1}, C_{2}$ such that

$$
y(t) \leq C_{1}(1+t)^{-2-\rho} \quad \text { and } \quad \int_{t}^{+\infty} y(\tau) \mathrm{d} \tau \leq C_{2}(1+t)^{-1-\rho}, \quad \forall t \geq 0 .
$$

Let us introduce the auxiliary energy functional

$$
\widetilde{\mathcal{E}}(t)=\mathcal{E}(\phi(t), \sigma(t))+K y(t)+\int_{t}^{+\infty} y(\tau) \mathrm{d} \tau
$$

It follows from (3.39) that, for a.e. $t \geq \delta$,

$$
\frac{\mathrm{d}}{\mathrm{d} t} \widetilde{\mathcal{E}}(t)+\mathcal{D}(t)^{2} \leq 0
$$


where

$$
\mathcal{D}(t)^{2}=\|\nabla \mu(t)\|_{L^{2}(\Omega)}^{2}+\|\nabla \sigma(t)\|_{L^{2}(\Omega)}^{2}+\int_{\Omega} P(\phi(t))(\mu(t)-\sigma(t))^{2} \mathrm{~d} x+y(t) .
$$

Hence, $\widetilde{\mathcal{E}}(t)$ is non-increasing in $t$. Since $\widetilde{\mathcal{E}}(t)$ is also bounded from below by its definition (3.42), we deduce that there exists a constant $\mathcal{E}_{\infty}$ such that

$$
\lim _{t \rightarrow+\infty} \widetilde{\mathcal{E}}(t)=\mathcal{E}_{\infty}
$$

which together with the decay property (3.41) yields

$$
\lim _{t \rightarrow+\infty} \mathcal{E}(\phi(t), \sigma(t))=\mathcal{E}_{\infty}
$$

Recalling the definition of $\omega\left(\phi_{0}, \sigma_{0}\right)$, it is easy to see that $\mathcal{E}(\phi(t), \sigma(t))$ equals to the constant $\mathcal{E}_{\infty}$ on the set $\omega\left(\phi_{0}, \sigma_{0}\right)$.

Step 2. Convergence of the trajectory $(\phi(t), \sigma(t))$. We proceed to prove that $\omega\left(\phi_{0}, \sigma_{0}\right)$ is actually a singleton. Since the constant limit $\sigma_{\infty}$ can be uniquely determined by $\phi_{\infty}$ via the mass constraint in (3.3) and $\mu_{\infty}$ can be uniquely determined by $\phi_{\infty}$ via (3.5), we only need to prove the uniqueness of asymptotic limit of the phase function $\phi(t)$ as $t \rightarrow+\infty$.

The proof of convergence of $\phi(t)$ follows from the so-called Lojasiewicz-Simon approach [53] (see e.g., [8, 23, 34, 40, 52] for its various aplications). To this end, we set

$$
m_{u}(t)=|\Omega|^{-1} \int_{t}^{+\infty} \int_{\Omega} u(\tau) \mathrm{d} x \mathrm{~d} \tau, \quad \forall t \geq 0 .
$$

Then it follows that

$$
\left|m_{u}(t)\right| \leq|\Omega|^{-\frac{1}{2}} \int_{t}^{+\infty}\|u(\tau)\|_{L^{2}(\Omega)} \mathrm{d} \tau=|\Omega|^{-\frac{1}{2}} y(t),
$$

where we recall (3.40) for the definition of $y(t)$.

For every element $\left(\phi_{\infty}, \sigma_{\infty}\right) \in \omega\left(\phi_{0}, \sigma_{0}\right)$, by Lemma 3.1 (with $m_{u}(t)$ given by (3.46) and allowing $t \in[0,+\infty)$ in $\left.m_{u}(t)\right)$, there exist constants $\theta_{\phi_{\infty}} \in\left(0, \frac{1}{2}\right)$ and $\beta_{\phi_{\infty}}>0$ such that the inequality (3.38) holds for $(\phi, \sigma) \in H_{N}^{2}(\Omega) \times H^{1}(\Omega)$ satisfying (3.37) and

$$
\phi \in \mathbf{B}_{\beta_{\phi_{\infty}}}\left(\phi_{\infty}\right):=\left\{\phi \in H_{N}^{2}(\Omega):\left\|\phi-\phi_{\infty}\right\|_{H^{1}(\Omega)}<\beta_{\phi_{\infty}}\right\} .
$$

The union of balls $\left\{\mathbf{B}_{\beta_{\phi_{\infty}}}\left(\phi_{\infty}\right):\left(\phi_{\infty}, \sigma_{\infty}\right) \in \omega\left(\phi_{0}, \sigma_{0}\right)\right\}$ forms an open cover of the set $\Phi:=\left\{\phi_{\infty}\right.$ : $\left.\left(\phi_{\infty}, \sigma_{\infty}\right) \in \omega\left(\phi_{0}, \sigma_{0}\right)\right\}$. Recalling (3.27) and (3.28), $\omega\left(\phi_{0}, \sigma_{0}\right)$ is compact in $H^{2}(\Omega) \times L^{2}(\Omega)$, thus we can find a finite sub-cover $\left\{\mathbf{B}_{\beta_{i}}\left(\phi_{\infty}^{i}\right): i=1,2, \ldots, m\right\}$ of $\Phi$ (in the topology of $H^{1}(\Omega)$ ), where the constants $\beta_{i}, \theta_{i}$ corresponding to $\phi_{\infty}^{i}$ in Lemma 3.1 are indexed by $i$. From the definition of $\omega\left(\phi_{0}, \sigma_{0}\right)$, there exists a sufficient large time $t_{0}>\delta$ such that

$$
\phi(t) \in \mathcal{U}:=\bigcup_{i=1}^{m} \mathbf{B}_{\beta_{i}}\left(\phi_{\infty}^{i}\right), \quad \text { for } t \geq t_{0}
$$

Taking $\theta=\min _{i=1}^{m}\left\{\theta_{i}\right\} \in\left(0, \frac{1}{2}\right)$, using Lemma 3.1. the convergence of energy (3.45) and the estimate (3.47), we deduce that, for all $t \geq t_{0}$, the global weak solution $(\phi(t), \sigma(t))$ satisfies

$$
\begin{aligned}
& \|\mu(t)-\bar{\mu}(t)\|_{\left(H^{1}(\Omega)\right)^{\prime}}+C\|\nabla \sigma(t)\|_{L^{2}(\Omega)}+C\|\sqrt{P(\phi(t))}(\mu(t)-\sigma(t))\|_{L^{2}(\Omega)}+C y(t)^{\frac{1}{2}} \\
& \quad \geq\left|\mathcal{E}(\phi(t), \sigma(t))-\mathcal{E}_{\infty}\right|^{1-\theta},
\end{aligned}
$$


which together with Poincaré's inequality and (3.26) yields

$$
\begin{aligned}
& C\|\nabla \mu(t)\|_{L^{2}(\Omega)}+C\|\nabla \sigma(t)\|_{L^{2}(\Omega)}+C\|\sqrt{P(\phi(t))}(\mu(t)-\sigma(t))\|_{L^{2}(\Omega)}+C y(t)^{\frac{1}{2}} \\
& \quad \geq\left|\mathcal{E}(\phi(t), \sigma(t))-\mathcal{E}_{\infty}\right|^{1-\theta} .
\end{aligned}
$$

It follows from (3.41) $-(3.43)$ that

$$
\begin{aligned}
\mathcal{E}(\phi(t), \sigma(t))-\mathcal{E}_{\infty} & \geq \int_{t}^{\infty} \mathcal{D}(\tau)^{2} \mathrm{~d} \tau-K y(t)-\int_{t}^{+\infty} y(\tau) \mathrm{d} \tau \\
& \geq \int_{t}^{\infty} \mathcal{D}(\tau)^{2} \mathrm{~d} \tau-C(1+t)^{-(1+\rho)}
\end{aligned}
$$

On the other hand, set

$$
\zeta=\min \left\{\theta, \frac{\rho}{2(1+\rho)}\right\} \in\left(0, \frac{1}{2}\right) .
$$

Then on account of (3.48) and the uniform estimates (3.26)-(3.28), we get

$$
\begin{aligned}
& \left|\mathcal{E}(\phi(t), \sigma(t))-\mathcal{E}_{\infty}\right| \\
& \leq\left[C\|\nabla \mu(t)\|_{L^{2}(\Omega)}+C\|\nabla \sigma(t)\|_{L^{2}(\Omega)}+C\|\sqrt{P(\phi(t))}(\mu(t)-\sigma(t))\|_{L^{2}(\Omega)}+C y(t)^{\frac{1}{2}}\right]^{\frac{1}{1-\theta}} \\
& \leq C\left[\|\nabla \mu(t)\|_{L^{2}(\Omega)}+\|\nabla \sigma(t)\|_{L^{2}(\Omega)}+\|\sqrt{P(\phi(t))}(\mu(t)-\sigma(t))\|_{L^{2}(\Omega)}+y(t)^{\frac{1}{2}}\right]^{\frac{1}{1-\zeta}} \\
& \leq C \mathcal{D}(t)^{\frac{1}{1-\zeta}}, \quad \forall t \geq t_{0} .
\end{aligned}
$$

It is also easy to verify that

$$
\int_{t}^{\infty}(1+\tau)^{-2(1+\rho)(1-\zeta)} \mathrm{d} \tau \leq \int_{t}^{\infty}(1+\tau)^{-(2+\rho)} \mathrm{d} \tau \leq(1+t)^{-(1+\rho)}, \quad \forall t \geq t_{0} .
$$

Hence, denote

$$
\mathcal{Z}(t)=\mathcal{D}(t)+(1+t)^{-(1+\rho)(1-\zeta)} .
$$

We infer from (3.49)-(3.51) that

$$
\int_{t}^{\infty} \mathcal{Z}(\tau)^{2} \mathrm{~d} \tau \leq C \mathcal{D}(t)^{\frac{1}{1-\zeta}}+C(1+t)^{-(1+\rho)} \leq C \mathcal{Z}(t)^{\frac{1}{1-\zeta}}, \quad \forall t \geq t_{0} .
$$

Recall the following lemma (see [23,40])

Lemma 3.2. Let $\zeta \in\left(0, \frac{1}{2}\right)$. Assume that $Z \geq 0$ be a measurable function on $(\tau,+\infty), Z \in$ $L^{2}(\tau,+\infty)$ and there exist $C>0$ and $t_{0} \geq \tau$ such that

$$
\int_{t}^{\infty} Z(s)^{2} \mathrm{~d} s \leq C Z(t)^{\frac{1}{1-\zeta}}, \quad \text { for a.e. } t \geq t_{0} .
$$

Then $Z \in L^{1}\left(t_{0},+\infty\right)$.

Then from (3.52) and Lemma 3.2, we can deduce that

$$
\int_{t_{0}}^{+\infty} \mathcal{Z}(t) \mathrm{d} t<+\infty
$$


which together with (3.20) yields

$$
\int_{t_{0}}^{+\infty}\left\|\phi_{t}\right\|_{\left(H^{1}(\Omega)\right)^{\prime}} \mathrm{d} t<+\infty
$$

As a consequence, the trajectory $\phi(t)$ converges strongly in $\left(H^{1}(\Omega)\right)^{\prime}$ as $t \rightarrow+\infty$. Thanks to the compactness of the trajectory in $H^{2}(\Omega) \times L^{2}(\Omega)$ for $t \geq t_{0}$, we can conclude from Corollary 3.1 that there exists a steady state $\left(\phi_{\infty}, \sigma_{\infty}\right) \in \omega\left(\phi_{0}, \sigma_{0}\right)$ such that

$$
\lim _{t \rightarrow+\infty}\left(\left\|\phi(t)-\phi_{\infty}\right\|_{H^{2}(\Omega)}+\left\|\sigma(t)-\sigma_{\infty}\right\|_{L^{2}(\Omega)}\right)=0 .
$$

Namely, $\omega\left(\phi_{0}, \sigma_{0}\right)$ consists of only one point $\left(\phi_{\infty}, \sigma_{\infty}\right)$. Then $\mu_{\infty}$ is also uniquely determined as well.

Step 3. Convergence rate. It follows from (3.48) and (3.41) (3.44) that

$$
\begin{aligned}
\left(\widetilde{\mathcal{E}}(t)-\mathcal{E}_{\infty}\right)^{2(1-\theta)} & \leq C \mathcal{D}(t)^{2}+C(1+t)^{-2(1-\theta)(1+\rho)} \\
& \leq-C \frac{d}{d t}\left(\widetilde{\mathcal{E}}(t)-\mathcal{E}_{\infty}\right)+C(1+t)^{-2(1-\theta)(1+\rho)}
\end{aligned}
$$

Then, by [4, Lemma 2.8], we obtain the decay rate of energy:

$$
\widetilde{\mathcal{E}}(t)-\mathcal{E}_{\infty} \leq C(1+t)^{-\kappa}, \quad \forall t \geq t_{0},
$$

with the exponent $\kappa$ given by

$$
\kappa=\min \left\{\frac{1}{1-2 \theta}, 1+\rho\right\} .
$$

From (3.43) we infer, for any $t \geq t_{0}$,

$$
\int_{t}^{2 t} \mathcal{D}(\tau) \mathrm{d} \tau \leq t^{\frac{1}{2}}\left(\int_{t}^{2 t} \mathcal{D}(\tau)^{2} \mathrm{~d} \tau\right)^{\frac{1}{2}} \leq C t^{\frac{1}{2}}\left(\widetilde{\mathcal{E}}(t)-\mathcal{E}_{\infty}\right)^{\frac{1}{2}} \leq C(1+t)^{\frac{1-\kappa}{2}} .
$$

Thus,

$$
\int_{t}^{+\infty} \mathcal{D}(\tau) \mathrm{d} \tau \leq \sum_{j=0}^{+\infty} \int_{2^{j} t}^{2^{j+1} t} \mathcal{D}(\tau) \mathrm{d} \tau \leq C \sum_{j=0}^{+\infty}\left(2^{j} t\right)^{-\lambda} \leq C(1+t)^{-\lambda}, \quad \forall t \geq t_{0}
$$

where

$$
\lambda=\frac{\kappa-1}{2}=\min \left\{\frac{\theta}{1-2 \theta}, \frac{\rho}{2}\right\}>0 .
$$

Therefore, it holds (cf. (3.20)

$$
\int_{t}^{+\infty}\left\|\phi_{t}(\tau)\right\|_{\left(H^{1}(\Omega)\right)^{\prime}} \mathrm{d} \tau \leq C \int_{t}^{+\infty} \mathcal{D}(\tau) \mathrm{d} \tau \leq C(1+t)^{-\lambda}, \quad \forall t \geq t_{0},
$$

which yields the convergence rate of $\phi$ in $\left(H^{1}(\Omega)\right)^{\prime}$ such that

$$
\left\|\phi(t)-\phi_{\infty}\right\|_{\left(H^{1}(\Omega)\right)^{\prime}} \leq C(1+t)^{-\lambda}, \quad \forall t \geq t_{0} .
$$

Integrating (1.1) over $\Omega$, and then integrating from $t$ to $+\infty$, we deduce the convergence rate of the mean value

$$
\left|\overline{\phi(t)}-\overline{\phi_{\infty}}\right|=\left|\int_{t}^{+\infty} \overline{\phi_{t}(\tau)} \mathrm{d} \tau\right|
$$




$$
\begin{aligned}
& =\left|\int_{t}^{+\infty} \overline{P(\phi(\tau))(\sigma(t)-\mu(t))} \mathrm{d} \tau\right| \\
& \leq C\|\sqrt{P(\phi(t))}\|_{L^{\infty}\left(t,+\infty ; L^{2}(\Omega)\right)} \int_{t}^{+\infty}\left(\int_{\Omega} P(\phi(\tau))(\sigma(\tau)-\mu(\tau))^{2} \mathrm{~d} x\right)^{\frac{1}{2}} \mathrm{~d} \tau \\
& \leq C \int_{t}^{+\infty} \mathcal{D}(\tau) \mathrm{d} \tau \\
& \leq C(1+t)^{-\lambda}, \quad \forall t \geq t_{0} .
\end{aligned}
$$

In a similar manner, we have

$$
\begin{aligned}
\left|\overline{\sigma(t)}-\sigma_{\infty}\right| & \leq C \int_{t}^{+\infty} \mathcal{D}(\tau) \mathrm{d} \tau+C \int_{t}^{+\infty}\|u(\tau)\|_{L^{2}(\Omega)} \mathrm{d} \tau \\
& \leq C(1+t)^{-\lambda}, \quad \forall t \geq t_{0}
\end{aligned}
$$

We now proceed to prove higher-order estimates using the energy method (see e.g., [34,60]). We just need to work with $t \geq t_{0}$ such that the uniform estimates (3.26)-(3.28) are available. Set the differences of functions

$$
\hat{\phi}(t)=\phi(t)-\phi_{\infty}, \quad \hat{\sigma}(t)=\sigma(t)-\sigma_{\infty}, \quad \hat{\mu}(t)=\mu(t)-\mu_{\infty} .
$$

Then the triple $(\hat{\phi}, \hat{\sigma}, \hat{\mu})$ satisfies the following system

$$
\begin{aligned}
& \hat{\phi}_{t}-\Delta \hat{\mu}=P(\phi)(\hat{\sigma}-\hat{\mu}), \\
& \hat{\mu}=-\Delta \hat{\phi}+F^{\prime}(\phi)-F^{\prime}\left(\phi_{\infty}\right), \\
& \hat{\sigma}_{t}-\Delta \hat{\sigma}=-P(\phi)(\hat{\sigma}-\hat{\mu})+u, \\
& \partial_{\nu} \hat{\phi}=\partial_{\nu} \hat{\mu}=\partial_{\nu} \hat{\sigma}=0, \\
& \left.\hat{\phi}\right|_{t=0}=\phi_{0}-\phi_{\infty},\left.\quad \hat{\sigma}\right|_{t=0}=\sigma_{0}-\sigma_{\infty} .
\end{aligned}
$$

Testing (3.58) by $\hat{\mu}$, (3.60) by $\hat{\sigma}$, respectively, then adding the resultants together, we have

$$
\begin{aligned}
\frac{\mathrm{d}}{\mathrm{d} t}( & \left.\frac{1}{2}\|\nabla \hat{\phi}\|_{L^{2}(\Omega)}^{2}+\int_{\Omega}\left(F(\phi)-F\left(\phi_{\infty}\right)+F^{\prime}\left(\phi_{\infty}\right) \phi_{\infty}-F^{\prime}\left(\phi_{\infty}\right) \phi\right) \mathrm{d} x+\frac{1}{2}\|\hat{\sigma}\|_{L^{2}(\Omega)}^{2}\right) \\
& +\|\nabla \hat{\mu}\|_{L^{2}(\Omega)}^{2}+\|\nabla \hat{\sigma}\|_{L^{2}(\Omega)}^{2}+\int_{\Omega} P(\phi)(\hat{\sigma}-\hat{\mu})^{2} \mathrm{~d} x \\
\quad= & \int_{\Omega} u \hat{\sigma} \mathrm{d} x:=I_{1} .
\end{aligned}
$$

Testing (3.58) by $A^{-1}(\hat{\phi}-\overline{\hat{\phi}})$, we get

$$
\begin{aligned}
\frac{1}{2} \frac{\mathrm{d}}{\mathrm{d} t}\|\hat{\phi}-\overline{\hat{\phi}}\|_{\left(H^{1}(\Omega)\right)^{\prime}}^{2}+\|\nabla \hat{\phi}\|_{L^{2}(\Omega)}^{2} & \\
= & -\int_{\Omega}\left(F^{\prime}(\phi)-F^{\prime}\left(\phi_{\infty}\right)\right) \hat{\phi} \mathrm{d} x+\overline{\hat{\phi}} \int_{\Omega}\left(F^{\prime}(\phi)-F^{\prime}\left(\phi_{\infty}\right)\right) \mathrm{d} x \\
& +\int_{\Omega} P(\phi)(\hat{\sigma}-\hat{\mu}) A^{-1}(\hat{\phi}-\overline{\hat{\phi}}) \mathrm{d} x \\
& :=I_{2}+I_{3}+I_{4} .
\end{aligned}
$$

Using Poincaré's inequality and Young's inequality, $I_{1}$ can be estimated as

$$
I_{1} \leq\|u\|_{L^{2}(\Omega)}\left(\|\hat{\sigma}-\overline{\hat{\sigma}}\|_{L^{2}(\Omega)}+\left\|\bar{\sigma}-\overline{\sigma_{\infty}}\right\|_{L^{2}(\Omega)}\right)
$$




$$
\begin{aligned}
& \leq \frac{1}{2}\|\nabla \hat{\sigma}\|_{L^{2}(\Omega)}^{2}+C\|u\|_{L^{2}(\Omega)}^{2}+C\left|\bar{\sigma}-\overline{\sigma_{\infty}}\right|^{2} \\
& \leq \frac{1}{2}\|\nabla \hat{\sigma}\|_{L^{2}(\Omega)}^{2}+C(1+t)^{-2 \lambda} .
\end{aligned}
$$

By the Newton-Leibniz formula

$$
F(\phi)=F\left(\phi_{\infty}\right)+F^{\prime}\left(\phi_{\infty}\right)\left(\phi-\phi_{\infty}\right)+\int_{0}^{1} \int_{0}^{1} F^{\prime \prime}\left(s z \phi+(1-s z) \phi_{\infty}\right) z\left(\phi-\phi_{\infty}\right)^{2} \mathrm{~d} s \mathrm{~d} z
$$

we deduce from (3.55) and (3.56) that

$$
\begin{aligned}
& \left|\int_{\Omega}\left(F(\phi)-F\left(\phi_{\infty}\right)+F^{\prime}\left(\phi_{\infty}\right) \phi_{\infty}-F^{\prime}\left(\phi_{\infty}\right) \phi\right) \mathrm{d} x\right| \\
& \quad=\left|\int_{\Omega} \int_{0}^{1} \int_{0}^{1} F^{\prime \prime}\left(s z \phi+(1-s z) \phi_{\infty}\right) z \hat{\phi}^{2} \mathrm{~d} s \mathrm{~d} z \mathrm{~d} x\right| \\
& \leq \max _{s, z \in[0,1]}\left\|F^{\prime \prime}\left(s z \phi+(1-s z) \phi_{\infty}\right)\right\|_{L^{\infty}(\Omega)}\|\hat{\phi}\|_{L^{2}(\Omega)}^{2} \\
& \leq C\|\hat{\phi}\|_{L^{2}(\Omega)}^{2} \\
& \leq C\|\nabla \hat{\phi}\|_{L^{2}}\|\hat{\phi}-\overline{\hat{\phi}}\|_{\left(H^{1}(\Omega)\right)^{\prime}}+C|\overline{\hat{\phi}}|^{2} \\
& \leq \frac{1}{4}\|\nabla \hat{\phi}\|_{L^{2}(\Omega)}^{2}+C(1+t)^{-2 \lambda}, \quad \forall t \geq t_{0} .
\end{aligned}
$$

In a similar manner, we have

$$
I_{2}+I_{3} \leq \frac{1}{4}\|\nabla \hat{\phi}\|_{L^{2}(\Omega)}^{2}+C(1+t)^{-2 \lambda}, \quad \forall t \geq t_{0} .
$$

Finally, for $I_{4}$, it holds

$$
\begin{aligned}
I_{4} & \leq\|\sqrt{P(\phi)}\|_{L^{\infty}(\Omega)}\|\sqrt{P(\phi)}(\hat{\sigma}-\hat{\mu})\|_{L^{2}(\Omega)}\|\hat{\phi}-\overline{\hat{\phi}}\|_{\left(H^{1}(\Omega)\right)^{\prime}} \\
& \leq \frac{1}{4} \int_{\Omega} P(\phi)(\hat{\sigma}-\hat{\mu})^{2} \mathrm{~d} x+C(1+t)^{-2 \lambda}, \quad \forall t \geq t_{0} .
\end{aligned}
$$

Denote

$$
\begin{aligned}
\mathcal{Y}(t)= & \frac{1}{2}\|\nabla \hat{\phi}\|_{L^{2}(\Omega)}^{2}+\int_{\Omega}\left(F(\phi)-F\left(\phi_{\infty}\right)+F^{\prime}\left(\phi_{\infty}\right) \phi_{\infty}-F^{\prime}\left(\phi_{\infty}\right) \phi\right) \mathrm{d} x \\
& +\frac{1}{2}\|\hat{\sigma}\|_{L^{2}(\Omega)}^{2}+\frac{1}{2}\|\hat{\phi}-\overline{\hat{\phi}}\|_{\left(H^{1}(\Omega)\right)^{\prime}}^{2} .
\end{aligned}
$$

It follows that

$$
\begin{cases}\mathcal{Y}(t) \geq C_{3}\left(\|\nabla \hat{\phi}(t)\|_{L^{2}(\Omega)}^{2}+\|\hat{\sigma}(t)\|_{L^{2}(\Omega)}^{2}\right)-C_{4}(1+t)^{-2 \lambda}, & \forall t \geq t_{0}, \\ \mathcal{Y}(t) \leq C_{3}^{\prime}\left(\|\nabla \hat{\phi}(t)\|_{L^{2}(\Omega)}^{2}+\|\hat{\sigma}(t)\|_{L^{2}(\Omega)}^{2}\right)+C_{4}^{\prime}(1+t)^{-2 \lambda}, & \forall t \geq t_{0},\end{cases}
$$

and from (3.63), (3.64), we have

$$
\frac{d}{d t} \mathcal{Y}(t)+C_{5} \mathcal{Y}(t) \leq C_{6}(1+t)^{-2 \lambda}
$$

From the above inequality, we can easily obtain the decay estimate (cf. e.g., [60])

$$
\mathcal{Y}(t) \leq C(1+t)^{-2 \lambda}, \quad \forall t \geq t_{0}
$$


The above estimate together with (3.56), (3.68) and Poincaré's inequality yields that

$$
\|\hat{\phi}(t)\|_{H^{1}(\Omega)}+\|\hat{\sigma}(t)\|_{L^{2}(\Omega)} \leq C(1+t)^{-\lambda}, \quad \forall t \geq t_{0} .
$$

Finally, by the higher-order estimate (3.28) and interpolation, we have

$$
\begin{aligned}
\|\hat{\mu}(t)\|_{L^{2}(\Omega)} & \leq\|\Delta \hat{\phi}(t)\|_{L^{2}(\Omega)}+\left\|F^{\prime}(\phi(t))-F^{\prime}\left(\phi_{\infty}\right)\right\|_{L^{2}(\Omega)} \\
& \leq\|\nabla \Delta \hat{\phi}(t)\|_{L^{2}(\Omega)}^{\frac{1}{2}}\|\nabla \hat{\phi}(t)\|_{L^{2}(\Omega)}^{\frac{1}{2}}+\left\|F^{\prime \prime}\right\|_{L^{3}(\Omega)}\|\hat{\phi}(t)\|_{L^{6}(\Omega)} \\
& \leq C\|\hat{\phi}(t)\|_{H^{1}(\Omega)}^{\frac{1}{2}},
\end{aligned}
$$

which together with (3.71) gives the decay estimate (3.7).

The proof of Theorem 3.1 is complete.

\section{Lyapunov Stability with Zero Mass Source}

Within this section, we always assume $u=0$. Then from (3.8) it follows that the total mass of the system (1.1) - (1.5) is now conserved:

$$
\int_{\Omega}(\phi(t)+\sigma(t)) \mathrm{d} x=\int_{\Omega}\left(\phi_{0}+\sigma_{0}\right) \mathrm{d} x, \quad \forall t \geq 0 .
$$

Definition 4.1. Let $m \in \mathbb{R}$ be an arbitrary given constant. Set

$$
\mathcal{Z}_{m}=\left\{(\phi, \sigma) \in H^{1}(\Omega) \times L^{2}(\Omega): \int_{\Omega}(\phi+\sigma) \mathrm{d} x=|\Omega| m\right\} .
$$

Any $\left(\phi^{*}, \sigma^{*}\right) \in \mathcal{Z}_{m}$ is called a local energy minimizer of the total energy $\mathcal{E}(\phi, \sigma)$ defined in (1.6), if there exists a constant $\chi>0$ such that $\mathcal{E}\left(\phi^{*}, \sigma^{*}\right) \leq \mathcal{E}(\phi, \sigma)$, for all $(\phi, \sigma) \in \mathcal{Z}_{m}$ satisfying $\left\|\left(\phi-\phi^{*}, \sigma-\sigma^{*}\right)\right\|_{H^{1}(\Omega) \times L^{2}(\Omega)}<\chi$. If $\chi=+\infty$, then $\left(\phi^{*}, \sigma^{*}\right)$ is called a global energy minimizer of $\mathcal{E}(\phi, \sigma)$ in $\mathcal{Z}_{m}$.

The main result of this section reads as follows.

Theorem 4.1 (Lyapunov Stability). Assume that (F1), (F2), (P1), (P2) are satisfied and $u=0$. Given $m \in \mathbb{R}$, let $\left(\phi^{*}, \sigma^{*}\right)$ be a local energy minimizer of $\mathcal{E}(\phi, \sigma)$ in $\mathcal{Z}_{m}$. Then, for any $\epsilon>0$, there exists a constant $\eta \in(0,1)$ such that for arbitrary initial datum $\left(\phi_{0}, \sigma_{0}\right) \in\left(H_{N}^{2}(\Omega) \cap\right.$ $\left.H^{3}(\Omega)\right) \times H^{1}(\Omega)$ satisfying $\int_{\Omega}\left(\phi_{0}+\sigma_{0}\right) \mathrm{d} x=|\Omega| m$ and $\left\|\phi_{0}-\phi^{*}\right\|_{H^{1}(\Omega)}+\left\|\sigma_{0}-\sigma^{*}\right\|_{L^{2}(\Omega)} \leq \eta$, problem (1.1) -(1.5) admits a unique global strong solution $(\phi, \sigma)$ such that

$$
\left\|\phi(t)-\phi^{*}\right\|_{H^{1}(\Omega)}+\left\|\sigma(t)-\sigma^{*}\right\|_{L^{2}(\Omega)} \leq \epsilon, \quad \forall t \geq 0 .
$$

Namely, any local energy minimizer of $\mathcal{E}(\phi, \sigma)$ in $\mathcal{Z}_{m}$ is locally Lyapunov stable.

We first derive some properties for the critical point of $\mathcal{E}(\phi, \sigma)$ in $\mathcal{Z}_{m}$. For any given $m \in \mathbb{R}$, we consider the following problem for $(\phi, \mu, \sigma)$

$$
\begin{cases}-\Delta \phi+F^{\prime}(\phi)=\mu, & \text { in } \Omega, \\ \partial_{\nu} \phi=0, & \text { on } \partial \Omega, \\ \int_{\Omega}(\phi+\sigma) \mathrm{d} x=|\Omega| m, & \end{cases}
$$

where $\mu$ and $\sigma$ are constants given by

$$
\sigma=\mu=|\Omega|^{-1} \int_{\Omega} F^{\prime}(\phi) \mathrm{d} x
$$

Then we have 
Lemma 4.1. Let assumption $(\boldsymbol{F} 1)$ be satisfied.

(1) If $\left(\phi^{*}, \sigma^{*}\right) \in H_{N}^{2}(\Omega) \times \mathbb{R}$ is a strong solution to problem (4.4)-(4.5), then $\left(\phi^{*}, \sigma^{*}\right)$ is a critical point of $\mathcal{E}(\phi, \sigma)$ in $\mathcal{Z}_{m}$. Conversely, if $\left(\phi^{*}, \sigma^{*}\right)$ is a critical point of $\mathcal{E}(\phi, \sigma)$ in $\mathcal{Z}_{m}$, then $\phi^{*} \in H_{N}^{2}(\Omega), \sigma^{*} \in \mathbb{R}$ and they satisfy (4.4) (4.5).

(2) If $\left(\phi^{*}, \sigma^{*}\right)$ is a local energy minimizer of $\mathcal{E}(\phi, \sigma)$ in $\mathcal{Z}_{m}$, then $\left(\phi^{*}, \sigma^{*}\right)$ is a critical point of $\mathcal{E}(\phi, \sigma)$.

(3) The functional $\mathcal{E}(\phi, \sigma)$ has at least one minimizer $\left(\phi^{*}, \sigma^{*}\right) \in \mathcal{Z}_{m}$ such that

$$
\mathcal{E}\left(\phi^{*}, \sigma^{*}\right)=\inf _{(\phi, \sigma) \in \mathcal{Z}_{m}} \mathcal{E}(\phi, \sigma) .
$$

Proof. Consider the Lagrange function

$$
\mathcal{L}(\phi, \sigma, \mu)=\mathcal{E}(\phi, \sigma)-\mu\left(\int_{\Omega}(\phi+\sigma) \mathrm{d} x-|\Omega| m\right),
$$

where $\mu$ is a constant Lagrange multiplier for the mass constraint. For any $(\psi, \xi) \in H^{1}(\Omega) \times L^{2}(\Omega)$ satisfying the constraint $\int_{\Omega}(\psi+\xi) \mathrm{d} x=0$, we have

$$
\begin{aligned}
& \left.\frac{\mathrm{d}}{\mathrm{d} \varepsilon}\right|_{\varepsilon=0} \mathcal{L}\left(\phi^{*}+\varepsilon \psi, \sigma^{*}+\varepsilon \xi\right) \\
& \quad=\lim _{\varepsilon \rightarrow 0} \frac{\mathcal{L}\left(\phi^{*}+\varepsilon \psi, \sigma^{*}+\varepsilon \xi\right)-\mathcal{L}\left(\phi^{*}, \sigma^{*}\right)}{\varepsilon} \\
& =\int_{\Omega}\left(\nabla \phi^{*} \cdot \nabla \psi+F^{\prime}\left(\phi^{*}\right) \psi-\mu \psi\right) \mathrm{d} x+\int_{\Omega}\left(\sigma^{*}-\mu\right) \xi \mathrm{d} x .
\end{aligned}
$$

Hence, the critical point of $\mathcal{E}(\phi, \sigma)$ in $\mathcal{Z}_{m}$, denoted by $\left(\phi^{*}, \sigma^{*}\right)$, is a weak solution of problem (4.4) satisfying $\sigma^{*}=\mu^{*}=|\Omega|^{-1} \int_{\Omega} F^{\prime}\left(\phi^{*}\right) \mathrm{d} x$. Then by the elliptic regularity theory we have $\phi^{*} \in H_{N}^{2}(\Omega)$. Proof for other statements of the lemma is standard and is omitted here.

Proof of Theorem 4.1, Let $(\phi, \mu, \sigma)$ be a strong solution to problem (1.1)-(1.5). Using a similar argument like [33, Lemma 6.4], we have the following estimates

$$
\|\nabla \mu\|_{L^{2}(\Omega)} \leq\|\phi\|_{H^{3}(\Omega)}+\left\|F^{\prime \prime}(\phi)\right\|_{L^{\infty}(\Omega)}\|\nabla \phi\|_{L^{2}(\Omega)},
$$

and

$$
\|\phi\|_{H^{3}(\Omega)} \leq \bar{C}\left(\|\nabla \mu\|_{L^{2}(\Omega)}+\left\|F^{\prime \prime}(\phi)\right\|_{L^{\infty}(\Omega)}\|\phi\|_{H^{1}(\Omega)}+\|\phi\|_{H^{1}(\Omega)}\right)
$$

where the positive constant $\bar{C}$ only depends on $\Omega$. Next, recalling the definition of $\mathcal{A}(t)$ (see (3.21) ), we infer from (3.23) that

$$
\frac{\mathrm{d}}{\mathrm{d} t} \mathcal{A}(t)+\left\|\nabla \phi_{t}\right\|_{L^{2}(\Omega)}^{2}+\left\|\sigma_{t}\right\|_{L^{2}(\Omega)}^{2} \leq C_{1}\left(\mathcal{A}(t)^{2}+1\right),
$$

where the constant $C_{1}$ only depends on $\left\|\phi_{0}\right\|_{H^{1}(\Omega)},\left\|\sigma_{0}\right\|_{L^{2}(\Omega)}, \Omega$ and $\alpha_{6}$. Besides, from (2.7) we still have

$$
\int_{0}^{+\infty} \mathcal{A}(t) \mathrm{d} t \leq C_{2}
$$

where $C_{2}$ depends on $\left\|\phi_{0}\right\|_{H^{1}(\Omega)},\left\|\sigma_{0}\right\|_{L^{2}(\Omega)}, \Omega$ and $\alpha_{6}$.

Step 1. For given $m \in \mathbb{R}$, let $\left(\phi^{*}, \sigma^{*}\right)$ be a local energy minimizer of $\mathcal{E}(\phi, \sigma)$ in $\mathcal{Z}_{m}$. Then by Lemma 4.1, $\left(\phi^{*}, \sigma^{*}\right) \in H_{N}^{2}(\Omega) \times \mathbb{R}$. By the elliptic regularity theory and assumption (F1), it is 
easy to see that $\phi^{*} \in H^{3}(\Omega)$. We consider an initial datum $\left(\phi_{0}, \sigma_{0}\right) \in\left(H_{N}^{2}(\Omega) \cap H^{3}(\Omega)\right) \times H^{1}(\Omega)$ satisfying $\int_{\Omega}\left(\phi_{0}+\sigma_{0}\right) \mathrm{d} x=|\Omega| m$. Besides, we assume that

$$
\begin{aligned}
& \left\|\phi_{0}-\phi^{*}\right\|_{H^{1}(\Omega)}+\left\|\sigma_{0}-\sigma^{*}\right\|_{L^{2}(\Omega)} \leq \eta, \\
& \left\|\phi_{0}\right\|_{H^{3}(\Omega)} \leq M_{1}, \quad\left\|\sigma_{0}\right\|_{H^{1}(\Omega)} \leq M_{2},
\end{aligned}
$$

where $\eta \in(0,1]$ is a constant to be chosen later and $M_{1}, M_{2}>0$ are sufficiently large but fixed numbers. It follows that

$$
\left\|\phi_{0}\right\|_{H^{1}(\Omega)} \leq\left\|\phi^{*}\right\|_{H^{1}(\Omega)}+1, \quad\left\|\sigma_{0}\right\|_{L^{2}(\Omega)} \leq\left\|\sigma^{*}\right\|_{L^{2}(\Omega)}+1,
$$

and, as a consequence, we have

$$
\mathcal{E}\left(\phi_{0}, \sigma_{0}\right) \leq M_{3}
$$

where the constant $M_{3}>0$ depends on $\left\|\phi^{*}\right\|_{H^{1}(\Omega)},\left\|\sigma^{*}\right\|_{L^{2}(\Omega)}, \Omega, \alpha_{2}$ and $\alpha_{4}$.

Step 2. For the above initial datum $\left(\phi_{0}, \sigma_{0}\right)$, problem (1.1)-(1.5) admits a unique strong solution $(\phi(t), \sigma(t))$ on $[0,+\infty)$ (cf. Remark 3.1). We note that

$$
\mathcal{A}(0) \leq M_{4}
$$

where the constant $M_{4}>0$ depends on $M_{1}, M_{2}, \Omega, \alpha_{1}, \alpha_{2}$ and $\alpha_{4}$, but is independent of $\eta$. From (4.9)-(4.13), it follows that

$$
\mathcal{A}(t) \leq M_{5}, \quad \forall t \geq 0
$$

where the constant $M_{5}>0$ only depends on $M_{4},\left\|\phi^{*}\right\|_{H^{1}(\Omega)},\left\|\sigma^{*}\right\|_{L^{2}(\Omega)}, \Omega, \alpha_{1}, \alpha_{2}, \alpha_{4}$ and $\alpha_{6}$. Then from (4.8) we infer that

$$
\|\phi(t)\|_{H^{3}(\Omega)} \leq M_{6}, \quad\|\sigma(t)\|_{H^{1}(\Omega)} \leq M_{7}, \quad \forall t \geq 0,
$$

where the constants $M_{6}, M_{7}$ are again independent of $\eta$. The above estimates also imply the following estimate on energy difference

$$
\begin{aligned}
&\left|\mathcal{E}\left(\phi_{0}, \sigma_{0}\right)-\mathcal{E}(\phi(t), \sigma(t))\right| \\
& \leq \leq \frac{1}{2}\left\|\nabla\left(\phi(t)+\phi_{0}\right)\right\|_{L^{2}(\Omega)}\left\|\nabla\left(\phi(t)-\phi_{0}\right)\right\|_{L^{2}(\Omega)}+\left\|F^{\prime}\right\|_{L^{2}(\Omega)}\left\|\phi(t)-\phi_{0}\right\|_{L^{2}(\Omega)} \\
& \quad+\frac{1}{2}\left\|\sigma(t)+\sigma_{0}\right\|_{L^{2}(\Omega)}\left\|\sigma(t)-\sigma_{0}\right\|_{L^{2}(\Omega)} \\
& \quad \leq M_{8}\left(\left\|\phi(t)-\phi_{0}\right\|_{H^{1}(\Omega)}+\left\|\sigma(t)-\sigma_{0}\right\|_{L^{2}(\Omega)}\right),
\end{aligned}
$$

where $M_{8}>0$ depends on $\left\|\phi^{*}\right\|_{H^{1}(\Omega)},\left\|\sigma^{*}\right\|_{L^{2}(\Omega)}, \Omega, \alpha_{2}, \alpha_{4}$ and $\alpha_{6}$, but is independent of $\eta$.

Step 3. For any $\epsilon>0$, we choose

$$
\varpi=\min \{1, \epsilon, \chi, \beta\}>0,
$$

where $\chi$ is the constant in the definition of the local minimizer (see Definition 4.1) and $\beta$ is the constant associated with $\left(\phi^{*}, \sigma^{*}\right)$ given by the Łojasiewicz-Simon inequality (see Lemma 3.1. taking $u=0$ ). For any

$$
\eta \in\left(0, \frac{\varpi}{2}\right]
$$

we define

$$
T_{\eta}=\inf \left\{t>0, \quad\left\|\phi(t)-\phi^{*}\right\|_{H^{1}(\Omega)}+\left\|\sigma(t)-\sigma^{*}\right\|_{L^{2}(\Omega)} \geq \varpi\right\} .
$$


Then, by (4.11) and the fact $(\phi(t), \sigma(t)) \in C\left([0,+\infty) ; H^{1}(\Omega) \times L^{2}(\Omega)\right)$, we have $T_{\eta}>0$.

Step 4. Our aim is to prove that there exists a constant $\eta \in\left(0, \frac{\varpi}{2}\right]$ such that $T_{\eta}=+\infty$. The goal can be achieved by a contradiction argument. If this is not the case, then for all $\eta \in\left(0, \frac{\varpi}{2}\right]$, it holds $T_{\eta}<+\infty$. Here and after, we shall always exclude the trivial case such that there is a $t_{0} \in\left[0, T_{\eta}\right]$ and $\mathcal{E}\left(\phi\left(t_{0}\right), \sigma\left(t_{0}\right)\right)=\mathcal{E}\left(\phi^{*}, \sigma^{*}\right)$. In this case, by virtue of the energy identity (1.7) (with $u=0$ ), we have $\left\|\phi_{t}\right\|_{\left(H^{1}(\Omega)\right)^{\prime}}=\left\|\sigma_{t}\right\|_{\left(H^{1}(\Omega)\right)^{\prime}}=0$, for all $t \geq t_{0}$, and the evolution simply stops.

Next, we consider the case $\mathcal{E}(\phi(t), \sigma(t))>\mathcal{E}\left(\phi^{*}, \sigma^{*}\right)$, for $t \in\left[0, T_{\eta}\right]$. By the definition of $\varpi$, the energy identity (1.7) (with $u=0$ ) and Lemma 3.1 (with $m_{u}=0$ ), we see that on $\left[0, T_{\eta}\right]$ it holds

$$
\begin{aligned}
- & \frac{\mathrm{d}}{\mathrm{d} t}\left[\mathcal{E}(\phi(t), \sigma(t))-\mathcal{E}\left(\phi^{*}, \sigma^{*}\right)\right]^{\theta} \\
& =-\theta\left[\mathcal{E}(\phi(t), \sigma(t))-\mathcal{E}\left(\phi^{*}, \sigma^{*}\right)\right]^{\theta-1} \frac{\mathrm{d}}{\mathrm{d} t} \mathcal{E}(\phi(t), \sigma(t)) \\
& \geq C_{3} \theta \frac{\|\nabla \mu\|_{L^{2}(\Omega)}^{2}+\|\nabla \sigma\|_{L^{2}(\Omega)}^{2}+\|\sqrt{P(\phi)}(\mu-\sigma)\|_{L^{2}(\Omega)}^{2}}{\|\nabla \mu\|_{L^{2}(\Omega)}+\|\nabla \sigma\|_{L^{2}}+\|\sqrt{P(\phi)}(\mu-\sigma)\|_{L^{2}(\Omega)}} \\
& \geq C_{4} \theta\left(\|\nabla \mu\|_{L^{2}(\Omega)}+\|\nabla \sigma\|_{L^{2}(\Omega)}+\|\sqrt{P(\phi)}(\mu-\sigma)\|_{L^{2}(\Omega)}\right) \\
& \geq C_{5}\left(\left\|\phi_{t}\right\|_{\left(H^{1}(\Omega)\right)^{\prime}}+\left\|\sigma_{t}\right\|_{\left(H^{1}(\Omega)\right)^{\prime}}\right),
\end{aligned}
$$

where in the last inequality we used (3.20) for $\phi_{t}$ and a similar estimate for $\sigma_{t}$.

Thus, recalling that $\mathcal{E}(\phi(t), \sigma(t))$ is nonincreasing in time, by the choice of $\varpi$ (in particular, $\varpi \leq \chi)$ and $T_{\eta}$, we see from (4.18) that

$$
\begin{aligned}
\int_{0}^{T_{\eta}} & \left(\left\|\phi_{t}(t)\right\|_{\left(H^{1}(\Omega)\right)^{\prime}}+\left\|\sigma_{t}(t)\right\|_{\left(H^{1}(\Omega)\right)^{\prime}}\right) \mathrm{d} t \\
\leq & C_{5}^{-1}\left[\mathcal{E}\left(\phi_{0}, \sigma_{0}\right)-\mathcal{E}\left(\phi^{*}, \sigma^{*}\right)\right]^{\theta} \\
\leq & C_{6}\left(\left\|\phi_{0}-\phi^{*}\right\|_{H^{1}(\Omega)}^{\theta}+\left\|\sigma_{0}-\sigma^{*}\right\|_{L^{2}(\Omega)}^{\theta}\right)
\end{aligned}
$$

where $C_{6}$ depends on $C_{5},\left\|\phi^{*}\right\|_{H^{1}(\Omega)},\left\|\sigma^{*}\right\|_{L^{2}(\Omega)}, \theta, \Omega, \alpha_{2}, \alpha_{4}, \alpha_{6}$, but is independent of $\eta$. From (4.19), we deduce that

$$
\begin{aligned}
\left\|\phi\left(T_{\eta}\right)-\phi^{*}\right\|_{H^{1}(\Omega)}+\left\|\sigma\left(T_{\eta}\right)-\sigma^{*}\right\|_{L^{2}(\Omega)} & \\
\leq & \left\|\phi_{0}-\phi^{*}\right\|_{H^{1}(\Omega)}+\left\|\sigma_{0}-\sigma^{*}\right\|_{L^{2}(\Omega)}+\left\|\phi\left(T_{\eta}\right)-\phi_{0}\right\|_{H^{1}(\Omega)}+\left\|\sigma\left(T_{\eta}\right)-\sigma_{0}\right\|_{L^{2}(\Omega)} \\
\leq & \left\|\phi_{0}-\phi^{*}\right\|_{H^{1}(\Omega)}+\left\|\sigma_{0}-\sigma^{*}\right\|_{L^{2}(\Omega)}+C_{7}\left\|\phi\left(T_{\eta}\right)-\phi_{0}\right\|_{H^{3}(\Omega)}^{\frac{1}{2}}\left\|\phi\left(T_{\eta}\right)-\phi_{0}\right\|_{\left(H^{1}(\Omega)\right)^{\prime}}^{\frac{1}{2}} \\
& +C_{7}\left\|\sigma\left(T_{\eta}\right)-\sigma_{0}\right\|_{H^{1}(\Omega)}^{\frac{1}{2}}\left\|\sigma\left(T_{\eta}\right)-\sigma_{0}\right\|_{\left(H^{1}(\Omega)\right)^{\prime}}^{\frac{1}{2}} \\
\leq & \left\|\phi_{0}-\phi^{*}\right\|_{H^{1}(\Omega)}+\left\|\sigma_{0}-\sigma^{*}\right\|_{L^{2}(\Omega)} \\
& +C_{7}\left(M_{1}+M_{2}+M_{6}+M_{7}\right)^{\frac{1}{2}}\left[\left(\int_{0}^{T_{\eta}}\left\|\phi_{t}(t)\right\|_{\left(H^{1}(\Omega)\right)^{\prime}} \mathrm{d} t\right)^{\frac{1}{2}}+\left(\int_{0}^{T_{\eta}}\left\|\sigma_{t}(t)\right\|_{\left(H^{1}(\Omega)\right)^{\prime}} \mathrm{d} t\right)^{\frac{1}{2}}\right] \\
& \leq\left\|\phi_{0}-\phi^{*}\right\|_{H^{1}(\Omega)}+\left\|\sigma_{0}-\sigma^{*}\right\|_{L^{2}(\Omega)}+C_{8}\left(\left\|\phi_{0}-\phi^{*}\right\|_{H^{1}(\Omega)}^{\frac{\theta}{2}}+\left\|\sigma_{0}-\sigma^{*}\right\|_{L^{2}(\Omega)}^{\frac{\theta}{2}}\right) .
\end{aligned}
$$

Finally, choosing

$$
\eta=\min \left\{\frac{\varpi}{2},\left(\frac{\varpi}{8 C_{8}}\right)^{\frac{2}{\theta}}\right\},
$$


we infer that

$$
\left\|\phi\left(T_{\eta}\right)-\phi^{*}\right\|_{H^{1}(\Omega)}+\left\|\sigma\left(T_{\eta}\right)-\sigma^{*}\right\|_{L^{2}(\Omega)} \leq \frac{\varpi}{2}+\frac{\varpi}{4}<\varpi
$$

which leads a contradiction with the definition of $T_{\eta}$.

The proof of Theorem 4.1 is complete.

Remark 4.1. The result on long-time behavior derived in Theorem 3.1 can be applied to the global strong solution obtained in Theorem 4.1. Although it is still not obvious to identify the asymptotic limit $\left(\phi_{\infty}, \sigma_{\infty}\right)$, we are able to conclude that $\left(\phi_{\infty}, \sigma_{\infty}\right)$ also satisfies $\left\|\phi_{\infty}-\phi^{*}\right\|_{H^{1}(\Omega)}+$ $\left\|\sigma_{\infty}-\sigma^{*}\right\|_{L^{2}(\Omega)} \leq \epsilon$ thanks to (4.3). In particular, if $\left(\phi^{*}, \sigma^{*}\right)$ is an isolated local energy minimizer then it is indeed locally asymptotic stable.

\section{The Optimal Control Problem}

In this section we study the optimal control problem $(\mathbf{C P})$. Let $T \in(0,+\infty)$ be a fixed maximal time, $Q=\Omega \times(0, T)$ and $\Sigma=\Omega \times(0, T)$. We make for the remainder of this paper the following general assumptions on the data:

(C1) $\beta_{Q}, \beta_{\Omega}, \beta_{S}, \beta_{u}, \beta_{T}, \alpha_{Q}$ are nonnegative constants but not all zero.

(C2) $\phi_{Q} \in L^{2}(Q), \phi_{\Omega} \in L^{2}(\Omega), \sigma_{\Omega} \in L^{2}(\Omega), u_{\min } \in L^{\infty}(Q), u_{\max } \in L^{\infty}(Q)$, and $u_{\min } \leq u_{\max }$, a.e. in $Q$.

(C3) Let $R>0 . \mathcal{U}_{R}$ is an open set in $L^{2}(Q)$ such that $\mathcal{U}_{\text {ad }} \subset \mathcal{U}_{R}$ and $\|u\|_{L^{2}(Q)} \leq R$, for all $u \in \mathcal{U}_{R}$.

Remark 5.1. Let us mention the main differences between this problem and the ones considered in [15] and [31]. Here we generalize the problem of [15] by adding the dependence on the medication time $\tau$ in the cost functional $\mathcal{J}$. Moreover, we can consider the $\tau$-dependent terms in $\phi$ in the cost functional, which we were not able to handle in the previous paper [31], mainly due to the fact that the control $u$ here is imposed in the nutrient equation instead of in the phase equation. Due to this fact, we are able to enhance the regularity results on $\phi$ without affecting the regularity of the control u (cf. Proposition 5.3 below).

\section{$5.1 \quad$ Existence}

From Proposition 2.1 it follows that the control-to-state operator $\mathcal{S}$,

$$
u \mapsto \mathcal{S}(u):=(\phi, \mu, \sigma)
$$

is well-defined and Lipschitz continuous as a mapping from $\mathcal{U}_{R} \subset L^{2}(Q)$ into the following space (see [15, Remark 2])

$$
\left(L^{\infty}\left(0, T ;\left(H^{1}(\Omega)\right)^{\prime}\right) \cap L^{2}\left(0, T ; H^{1}(\Omega)\right)\right) \times L^{2}\left(0, T ;\left(H^{1}(\Omega)\right)^{\prime}\right) \times\left(L^{\infty}\left(0, T ;\left(H^{1}(\Omega)\right)^{\prime}\right) \cap L^{2}(Q)\right) .
$$

The triplet $(\phi, \mu, \sigma)$ is the unique weak solution to problem (1.1) (1.5) with data $\left(\phi_{0}, \sigma_{0}, u\right)$ over the time interval $[0, T]$. For convenience, we use the notations $\phi=\mathcal{S}_{1}(u)$ and $\sigma=\mathcal{S}_{3}(u)$ for the first and third component of $\mathcal{S}(u)$. Then we prove the following result that implies the existence of a solution to problem (CP). 
Theorem 5.1 (Existence of an optimal control). Assume that $(\boldsymbol{P} 1),(\boldsymbol{F} 1),(\boldsymbol{U} 1)$ and $(C 1)-$ (C3) are satisfied. Let $\phi_{0} \in H_{N}^{2}(\Omega) \cap H^{3}(\Omega)$ and $\sigma_{0} \in H^{1}(\Omega)$. Then there exists at least one minimizer $\left(\phi_{*}, \sigma_{*}, u_{*}, \tau_{*}\right)$ to problem $(\boldsymbol{C P})$. Namely, $\phi_{*}=\mathcal{S}_{1}\left(u_{*}\right), \sigma_{*}=\mathcal{S}_{3}\left(u_{*}\right)$ satisfy

$$
\mathcal{J}\left(\phi_{*}, \sigma_{*}, u_{*}, \tau_{*}\right)=\inf _{\substack{(w, s) \in \mathcal{U}_{\mathrm{ad}} \times[0, T] \\ \text { s.t. } \phi=\mathcal{S}_{1}(w), \sigma=\mathcal{S}_{3}(w)}} \mathcal{J}(\phi, \sigma, w, s) .
$$

Proof. As the cost functional $\mathcal{J}$ is bounded from below, we can find a minimizing sequence $\left(u_{n}, \tau_{n}\right)_{n \in \mathbb{N}}$ with $u_{n} \in \mathcal{U}_{\text {ad }}, \tau_{n} \in(0, T)$ and the corresponding weak solutions $\left(\phi_{n}, \mu_{n}, \sigma_{n}\right)_{n \in \mathbb{N}}$ on the interval $[0, T]$ with $\phi_{n}(0)=\phi_{0}$ and $\sigma_{n}(0)=\sigma_{0}$, for all $n \in \mathbb{N}$, such that

$$
\lim _{n \rightarrow+\infty} \mathcal{J}\left(\phi_{n}, \sigma_{n}, u_{n}, \tau_{n}\right)=\inf _{\substack{(w, s) \in \mathcal{U}_{\mathrm{ad}} \times[0, T] \\ \text { s.t. } \phi=\mathcal{S}_{1}(w), \sigma=\mathcal{S}_{3}(w)}} \mathcal{J}(\phi, \sigma, w, s) .
$$

In particular, $u_{n} \in \mathcal{U}_{\text {ad }}$ implies that $u_{n}$ is bounded in $L^{\infty}(Q)$ for all $n \in \mathbb{N}$. As $\left\{\tau_{n}\right\}_{n \in \mathbb{N}}$ is a bounded sequence, there exists a convergent subsequence still denoted by $\left\{\tau_{n}\right\}$ such that

$$
\tau_{n} \rightarrow \tau_{*} \in[0, T] \quad \text { as } n \rightarrow+\infty .
$$

Besides, arguing exactly as in [15, Section 4], we can find a further subsequence, which is again indexed by $n$, such that

$$
\begin{aligned}
& u_{n} \rightarrow u \quad \text { weakly star in } L^{\infty}(Q), \\
& \phi_{n} \rightarrow \phi \quad \text { weakly star in } H^{1}\left(0, T ; H^{1}(\Omega)\right) \cap L^{\infty}\left(0, T ; H^{3}(\Omega)\right), \\
& \Delta \phi_{n} \rightarrow \Delta \phi \quad \text { weakly in } L^{2}\left(0, T ; H_{N}^{2}(\Omega)\right), \\
& \mu_{n} \rightarrow \mu \quad \text { weakly star in } L^{\infty}\left(0, T ; H^{1}(\Omega)\right) \cap L^{2}\left(0, T ; H_{N}^{2}(\Omega)\right), \\
& \sigma_{n} \rightarrow \sigma \quad \text { weakly star in } H^{1}\left(0, T ; L^{2}(\Omega)\right) \cap L^{\infty}\left(0, T ; H^{1}(\Omega)\right) \cap L^{2}\left(0, T ; H_{N}^{2}(\Omega)\right),
\end{aligned}
$$

and, moreover,

$$
\phi_{n} \rightarrow \phi \quad \text { strongly in } C^{0}\left([0, T] ; H^{2}(\Omega)\right),
$$

which implies $\phi_{n} \rightarrow \phi$ strongly in $C^{0}(\bar{Q})$. Then we see that

$$
F^{\prime}\left(\phi_{n}\right) \rightarrow F^{\prime}(\phi) \text { and } P\left(\phi_{n}\right) \rightarrow P(\phi), \text { strongly in } C^{0}(\bar{Q}) .
$$

As a consequence, we are able to pass to the limit as $n \rightarrow \infty$ in problem (1.1)-(1.5) (written for $\left.\left(\phi_{n}, \mu_{n}, \sigma_{n}\right)\right)$, finding that $(\phi, \mu, \sigma)=\mathcal{S}(u)$. Namely, the pair $((\phi, \mu, \sigma), u)$ is admissible for the control problem (CP). Furthermore, by the dominating convergence theorem, for all $p \in[1, \infty)$, we have $\chi_{\left[0, \tau_{n}\right]}(t) \rightarrow \chi_{\left[0, \tau_{*}\right]}(t)$ strongly in $L^{p}(0, T)$. Then by the strong convergence of $\phi_{n}-\phi_{Q}$ to $\phi_{*}-\phi_{Q}$ in $L^{2}(Q)$ and the strong convergence $\chi_{\left[0, \tau_{n}\right]}(t)$ to $\chi_{\left[0, \tau_{*}\right]}(t)$ also in $L^{2}(Q)$, we deduce that, as $n \rightarrow \infty$,

$$
\begin{aligned}
\int_{0}^{\tau_{n}} \int_{\Omega}\left|\phi_{n}-\phi_{Q}\right|^{2} \mathrm{~d} x \mathrm{~d} t & =\int_{0}^{T}\left\|\phi_{n}-\phi_{Q}\right\|_{L^{2}(\Omega)}^{2} \chi_{\left[0, \tau_{n}\right]}(t) \mathrm{d} t \\
& \longrightarrow \int_{0}^{T}\left\|\phi_{*}-\phi_{Q}\right\|_{L^{2}(\Omega)}^{2} \chi_{\left[0, \tau^{*}\right]}(t) \mathrm{d} t \\
& =\int_{0}^{\tau_{*}} \int_{\Omega}\left|\phi_{*}-\phi_{Q}\right|^{2} \mathrm{~d} x \mathrm{~d} t .
\end{aligned}
$$

The convergence of other terms in $\mathcal{J}$ can be treated in similar manner. Hence, from the weak sequential lower semicontinuity property of $\mathcal{J}$, it follows that $\left(u_{*}, \tau_{*}\right)$ is indeed a minimizer of the control problem (CP).

The proof is complete. 


\subsection{Differentiability of the solution operator $\mathcal{S}$ with respect to $u$}

First, we establish the Fréchet differentiability of the solution operator $\mathcal{S}$ with respect to the control $u$. For this purpose, we investigate the linearized state equation. For arbitrary, but fixed $u_{*} \in \mathcal{U}_{R}$, let $\left(\phi_{*}, \mu_{*}, \sigma_{*}\right)=\mathcal{S}\left(u_{*}\right)$. We consider for any $h \in L^{2}(Q)$ the linearized system

$$
\begin{array}{ll}
\partial_{t} \xi-\Delta \eta=P^{\prime}\left(\phi_{*}\right)\left(\sigma_{*}-\mu_{*}\right) \xi+P\left(\phi_{*}\right)(\rho-\eta), & \text { in } Q, \\
\eta=-\Delta \xi+F^{\prime \prime}\left(\phi_{*}\right) \xi, & \text { in } Q, \\
\partial_{t} \rho-\Delta \rho=-P^{\prime}\left(\phi_{*}\right)\left(\sigma_{*}-\mu_{*}\right) \xi-P\left(\phi_{*}\right)(\rho-\eta)+h, & \text { in } Q, \\
\partial_{\mathbf{n}} \xi=\partial_{\mathbf{n}} \eta=\partial_{\mathbf{n}} \rho=0, & \text { on } \Sigma, \\
\xi(0)=\rho(0)=0, & \text { in } \Omega .
\end{array}
$$

Observe that the linearized system (5.2)-(5.6) is exactly the same as the one obtained in [15, Section 3]. Hence, we can simply quote [15, Theorems 3.1, 3.2] for the well-posedness of the linearized system (5.2) - (5.6) and the Fréchet differentiability of the control-to-state operator $\mathcal{S}$ with respect to $u$. More precisely, we have

Proposition 5.1. Assume that $(\boldsymbol{P} 1)$ and $(\boldsymbol{F} 1)$ are satisfied, $h \in L^{2}(Q)$. The system (5.2) -(5.6) admits a unique solution $(\xi, \eta, \rho)$ such that

$$
\begin{aligned}
& \xi \in H^{1}\left(0, T ;\left(H^{1}(\Omega)\right)^{\prime}\right) \cap L^{\infty}\left(0, T ; H^{1}(\Omega)\right) \cap L^{2}\left(0, T ; H_{N}^{2}(\Omega) \cap H^{3}(\Omega)\right), \\
& \eta \in L^{2}\left(0, T ; H^{1}(\Omega)\right), \quad \rho \in H^{1}\left(0, T ; L^{2}(\Omega)\right) \cap C^{0}\left([0, T] ; H^{1}(\Omega)\right) \cap L^{2}\left(0, T ; H_{N}^{2}(\Omega)\right) .
\end{aligned}
$$

The triple $(\xi, \eta, \rho)$ fulfills (5.3)-(5.6) almost everywhere in the respective sets, except for (5.2) and the related boundary condition in (5.5) that are satisfied, for almost every $t \in(0, T)$, in the following sense

$$
\begin{aligned}
& \left\langle\xi_{t}(t), v\right\rangle_{\left(H^{1}(\Omega)\right)^{\prime}, H^{1}(\Omega)}+\int_{\Omega} \nabla \eta(t) \cdot \nabla v \mathrm{~d} x \\
& \quad=\int_{\Omega} P^{\prime}\left(\phi_{*}(t)\right)\left(\sigma_{*}(t)-\mu_{*}(t)\right) \xi(t) v \mathrm{~d} x+\int_{\Omega} P\left(\phi_{*}(t)\right)(\rho(t)-\eta(t)) v \mathrm{~d} x, \quad \forall v \in H^{1}(\Omega) .
\end{aligned}
$$

Moreover, there exists some constant $K_{3}>0$, which depends only on $R$ and the data of the state system, such that

$$
\begin{aligned}
& \|\xi\|_{H^{1}\left(0, t ;\left(H^{1}(\Omega)\right)^{\prime}\right) \cap L^{\infty}\left(0, t ; H^{1}(\Omega)\right) \cap L^{2}\left(0, t ; H^{3}(\Omega)\right)}+\|\eta\|_{L^{2}\left(0, t ; H^{1}(\Omega)\right)} \\
& \quad+\|\rho\|_{H^{1}\left(0, t ; L^{2}(\Omega)\right) \cap C^{0}\left([0, t] ; H^{1}(\Omega)\right) \cap L^{2}\left(0, t ; H^{2}(\Omega)\right)} \leq K_{3}\|h\|_{L^{2}\left(0, t ; L^{2}(\Omega)\right)}, \quad \forall t \in[0, T] .
\end{aligned}
$$

Proposition 5.2. Assume that (P1), (F1), (U1) and (C1)-(C3) are satisfied. Let $\phi_{0} \in$ $H_{N}^{2}(\Omega) \cap H^{3}(\Omega)$ and $\sigma_{0} \in H^{1}(\Omega)$. Then the control-to-state operator $\mathcal{S}$ is Fréchet differentiable in $\mathcal{U}_{R}$ as a mapping from $L^{2}(Q)$ into the space

$$
\begin{aligned}
\mathcal{Y}:=( & \left.H^{1}\left(0, T ;\left(H_{N}^{2}(\Omega)\right)^{\prime}\right) \cap L^{\infty}\left(0, T ; L^{2}(\Omega)\right) \cap L^{2}\left(0, T ; H_{N}^{2}(\Omega)\right)\right) \times L^{2}(Q) \\
& \times\left(H^{1}\left(0, T ; L^{2}(\Omega)\right) \cap L^{2}\left(0, T ; H^{2}(\Omega)\right)\right) .
\end{aligned}
$$

Moreover, for any $u_{*} \in \mathcal{U}_{R}$, the Fréchet derivative $D \mathcal{S}\left(u_{*}\right) \in \mathcal{L}\left(L^{2}(Q), \mathcal{Y}\right)$ is defined as follows: for any $h \in L^{2}(Q)$, we have

$$
D \mathcal{S}\left(u_{*}\right) h=\left(\xi^{h}, \eta^{h}, \rho^{h}\right),
$$

where $\left(\xi^{h}, \eta^{h}, \rho^{h}\right)$ is the unique solution to the linearized system (5.2)-(5.6) associated with $h$. 


\subsection{The first order necessary optimality conditions}

Define a reduced functional

$$
\widetilde{\mathcal{J}}(u, \tau):=\mathcal{J}\left(S_{1}(u), S_{3}(u), u, \tau\right)
$$

Since the embedding of $H^{1}\left(0, T ;\left(H_{N}^{2}(\Omega)\right)^{\prime}\right) \cap L^{2}\left(0, T ; H_{N}^{2}(\Omega)\right)$ in $C^{0}\left([0, T] ; L^{2}(\Omega)\right)$ is continuous, from Proposition 5.2 it follows that the control-to-state mapping $\mathcal{S}$ is also Fréchet differentiable into $C^{0}\left([0, T] ; L^{2}(\Omega)\right)$ with respect to $u$. From this we deduce that the reduced cost functional $\widetilde{\mathcal{J}}$ is Fréchet differentiable in $\mathcal{U}_{R}$.

The first order necessary optimality conditions for the minimizer $\left(u_{*}, \tau_{*}\right)$ of Theorem 5.1 also requires the Fréchet derivative of $\mathcal{J}$ (or equivalently, $\widetilde{\mathcal{J}}$ ) with respect to $\tau$. For this purpose, we need a further regularity result on the state problem (1.1)-(1.5), under a stronger assumption on the initial data $\left(\phi_{0}, \sigma_{0}\right)$.

Proposition 5.3. Assume that (P1), (F1) and (U1) are satisfied. Let $\phi_{0} \in H_{N}^{2}(\Omega) \cap H^{6}(\Omega)$ and $\sigma_{0} \in H_{N}^{2}(\Omega)$. For every $T>0$, problem (1.1) - (1.5) admits a unique strong solution on $[0, T]$ such that the following extra regularity properties hold

$$
\phi \in H^{2}\left(0, T ; L^{2}(\Omega)\right), \quad \mu \in W^{1, \infty}\left(0, T ; L^{2}(\Omega)\right) .
$$

Moreover, there exists a constant $K_{4}>0$, depending on $\|u\|_{L^{2}\left(0, T ; L^{2}(\Omega)\right)}, \Omega,\left\|\phi_{0}\right\|_{H^{6}(\Omega)}$ and $\left\|\sigma_{0}\right\|_{H^{2}(\Omega)}$, such that

$$
\|\phi\|_{H^{2}\left(0, T ; L^{2}(\Omega)\right)}+\|\mu\|_{W^{1, \infty}\left(0, T ; L^{2}(\Omega)\right)} \leq K_{4} .
$$

Proof. We perform here formal a priori estimates which can be made rigorous by means of a standard approximation scheme. Testing the time derivative of (1.1) by $\phi_{t}$, summing it with the time derivative of (1.2) tested by $\mu_{t}$ and then integrating in time over $(0, T)$, from (2.8), Hölder's inequality and Young's inequality we infer that

$$
\begin{aligned}
\frac{1}{2}\left\|\phi_{t}(t)\right\|_{L^{2}(\Omega)}^{2}+\int_{0}^{T}\left\|\mu_{t}\right\|_{L^{2}(\Omega)}^{2} \mathrm{~d} t \\
\leq \frac{1}{2}\left\|\phi_{t}(0)\right\|_{L^{2}(\Omega)}^{2}+\int_{0}^{T}\left\|P^{\prime}(\phi)\right\|_{L^{\infty}(\Omega)}\left\|\phi_{t}\right\|_{L^{2}(\Omega)}^{2}\left(\|\sigma\|_{L^{\infty}(\Omega)}+\|\mu\|_{L^{\infty}(\Omega)}\right) \mathrm{d} t \\
\quad+\int_{0}^{T}\|P(\phi)\|_{L^{\infty}(\Omega)}\left(\left\|\sigma_{t}\right\|_{L^{2}(\Omega)}+\left\|\mu_{t}\right\|_{L^{2}(\Omega)}\right)\left\|\phi_{t}\right\|_{L^{2}(\Omega)} \mathrm{d} t \\
\quad+\int_{0}^{T}\left\|F^{\prime \prime}(\phi)\right\|_{L^{\infty}(\Omega)}\left\|\phi_{t}\right\|_{L^{2}(\Omega)}\left\|\mu_{t}\right\|_{L^{2}(\Omega)} \mathrm{d} t \\
\leq C+\frac{1}{2} \int_{0}^{T}\left\|\mu_{t}\right\|_{L^{2}(\Omega)}^{2} \mathrm{~d} t+C \int_{0}^{T}\left\|\sigma_{t}\right\|_{L^{2}(\Omega)}^{2} \mathrm{~d} t \\
\quad+C \int_{0}^{T}\left(1+\|\sigma\|_{H^{2}(\Omega)}+\|\mu\|_{H^{2}(\Omega)}\right)\left\|\phi_{t}\right\|_{L^{2}(\Omega)}^{2} \mathrm{~d} t \\
\leq C+\frac{1}{2} \int_{0}^{T}\left\|\mu_{t}\right\|_{L^{2}(\Omega)}^{2} \mathrm{~d} t+C \int_{0}^{T}\left(1+\|\sigma\|_{H^{2}(\Omega)}+\|\mu\|_{H^{2}(\Omega)}\right)\left\|\phi_{t}\right\|_{L^{2}(\Omega)}^{2} \mathrm{~d} t,
\end{aligned}
$$

where $C>0$ is a constant depending on $\left\|\phi_{0}\right\|_{H^{4}(\Omega)},\left\|\sigma_{0}\right\|_{H^{1}(\Omega)},\|u\|_{L^{2}\left(0, T ; L^{2}(\Omega)\right)}, \Omega$ and $T$. Using now estimate (2.8) and Gronwall's lemma, we conclude that

$$
\left\|\phi_{t}\right\|_{L^{\infty}\left(0, T ; L^{2}(\Omega)\right)}+\left\|\mu_{t}\right\|_{L^{2}\left(0, T ; L^{2}(\Omega)\right)} \leq C
$$

where $C>0$ is a constant depending on $\left\|\phi_{0}\right\|_{H^{4}(\Omega)},\left\|\sigma_{0}\right\|_{H^{1}(\Omega)},\|u\|_{L^{2}\left(0, T ; L^{2}(\Omega)\right)}, \Omega$ and $T$. 
Next, testing the time derivative of (1.1) by $\phi_{t t}$, summing it to the second time derivative of (1.2) tested by $\mu_{t}$ and then integrating over $(0, T)$, from (2.8), Hölder's inequality and Young's inequality we infer that

$$
\begin{aligned}
\frac{1}{2}\left\|\mu_{t}(t)\right\|_{L^{2}(\Omega)}^{2}+\int_{0}^{T}\left\|\phi_{t t}\right\|_{L^{2}(\Omega)}^{2} \mathrm{~d} t \\
\leq \frac{1}{2}\left\|\mu_{t}(0)\right\|_{L^{2}(\Omega)}^{2}+\int_{0}^{T}\left\|P^{\prime}(\phi)\right\|_{L^{\infty}(\Omega)}\left(\|\sigma\|_{L^{4}(\Omega)}+\|\mu\|_{L^{4}(\Omega)}\right)\left\|\phi_{t}\right\|_{L^{4}(\Omega)}\left\|\phi_{t t}\right\|_{L^{2}(\Omega)} \mathrm{d} t \\
\quad+\int_{0}^{T}\|P(\phi)\|_{L^{\infty}(\Omega)}\left(\left\|\sigma_{t}\right\|_{L^{2}(\Omega)}+\left\|\mu_{t}\right\|_{L^{2}(\Omega)}\right)\left\|\phi_{t t}\right\|_{L^{2}(\Omega)} \mathrm{d} t \\
\quad+\int_{0}^{T}\left\|F^{\prime \prime \prime}(\phi)\right\|_{L^{\infty}(\Omega)}\left\|\phi_{t}\right\|_{L^{4}(\Omega)}^{2}\left\|\mu_{t}\right\|_{L^{2}(\Omega)} \mathrm{d} t \\
\quad+\int_{0}^{T}\left\|F^{\prime \prime}(\phi)\right\|_{L^{\infty}(\Omega)}\left\|\phi_{t t}\right\|_{L^{2}(\Omega)}\left\|\mu_{t}\right\|_{L^{2}(\Omega)} \mathrm{d} t \\
\leq C+\frac{1}{2} \int_{0}^{T}\left\|\phi_{t t}\right\|_{L^{2}(\Omega)}^{2} \mathrm{~d} t+\int_{0}^{T}\left(\|\sigma\|_{L^{4}(\Omega)}^{2}+\|\mu\|_{L^{4}(\Omega)}^{2}\right)\left\|\phi_{t}\right\|_{L^{4}(\Omega)}^{2} \mathrm{~d} t \\
\quad+C \int_{0}^{T}\left(\left\|\sigma_{t}\right\|_{L^{2}(\Omega)}^{2}+\left\|\mu_{t}\right\|_{L^{2}(\Omega)}^{2}\right) \mathrm{d} t+C \int_{0}^{T}\left\|\phi_{t}\right\|_{L^{4}(\Omega)}^{2}\left(\left\|\mu_{t}\right\|_{L^{2}(\Omega)}^{2}+1\right) \mathrm{d} t \\
\leq C+\frac{1}{2} \int_{0}^{T}\left\|\phi_{t t}\right\|_{L^{2}(\Omega)}^{2} \mathrm{~d} t+C \int_{0}^{T}\left\|\phi_{t}\right\|_{H^{1}(\Omega)}^{2}\left\|\mu_{t}\right\|_{L^{2}(\Omega)}^{2} \mathrm{~d} t
\end{aligned}
$$

where $C>0$ is a constant depending on $\left\|\phi_{0}\right\|_{H^{6}(\Omega)},\left\|\sigma_{0}\right\|_{H^{1}(\Omega)},\|u\|_{L^{2}\left(0, T ; L^{2}(\Omega)\right)}, \Omega$ and $T$. Using estimate (2.8), from Gronwall's lemma we deduce

$$
\left\|\mu_{t}\right\|_{L^{\infty}\left(0, T ; L^{2}(\Omega)\right)}+\left\|\phi_{t t}\right\|_{L^{2}\left(0, T ; L^{2}(\Omega)\right)} \leq C
$$

where $C$ is a constant depending on $\left\|\phi_{0}\right\|_{H^{6}(\Omega)},\left\|\sigma_{0}\right\|_{H^{1}(\Omega)},\|u\|_{L^{2}\left(0, T ; L^{2}(\Omega)\right)}, \Omega$ and $T$. This concludes the proof of Proposition 5.3.

Taking advantage of the higher-order regularity result illustrated in Proposition 5.3 and using a similar argument like for [31, Theorem 2.6], we obtain

Proposition 5.4. Assume that $(\boldsymbol{P} 1),(\boldsymbol{F} 1),(\boldsymbol{U} 1)$ and $(C 1)-(C 3)$ are satisfied. For any $\phi_{0} \in H_{N}^{2}(\Omega) \cap H^{6}(\Omega), \sigma_{0} \in H^{1}(\Omega)$ and $u \in \mathcal{U}_{\mathrm{ad}}$, we denote the corresponding state variables by $\mathcal{S}(u)=(\phi, \mu, \sigma)$. Assume in addition that $\phi_{Q} \in H^{1}\left(0, T ; L^{2}(\Omega)\right)$. Then the reduced functional $\widetilde{\mathcal{J}}(u, \tau)$ is Fréchet differentiable with respect to $\tau$ and it holds

$$
\begin{aligned}
D_{\tau} \widetilde{\mathcal{J}}(u, \tau)= & \frac{\beta_{Q}}{2} \int_{\Omega}\left|\phi(\tau)-\phi_{Q}(\tau)\right|^{2} \mathrm{~d} x+\beta_{\Omega} \int_{\Omega}\left(\phi(\tau)-\phi_{\Omega}\right) \phi_{t}(\tau) \mathrm{d} x \\
& +\frac{\alpha_{Q}}{2} \int_{\Omega}\left|\sigma(\tau)-\sigma_{Q}\right|^{2} \mathrm{~d} x+\frac{\beta_{S}}{2} \int_{\Omega} \phi_{t}(\tau) \mathrm{d} x+\beta_{T} .
\end{aligned}
$$

Propositions 5.2 and 5.4 allow us to derive the following first order necessary optimality condition for problem $(\mathbf{C P})$.

Theorem 5.2 (First order necessary optimality condition). Assume that (P1), (F1), (U1), (C1)-(C3) are satisfied, $\phi_{0} \in H_{N}^{2}(\Omega) \cap H^{6}(\Omega), \sigma_{0} \in H^{1}(\Omega)$ and $\phi_{Q} \in H^{1}\left(0, T ; L^{2}(\Omega)\right)$. Suppose that $\left(u_{*}, \tau_{*}\right) \in \mathcal{U}_{\mathrm{ad}} \times[0, T]$ is solution to the control problem $(\mathbf{C P})$ with associated state $\left(\phi_{*}, \mu_{*}, \sigma_{*}\right)=\mathcal{S}\left(u_{*}\right)$. Then, it holds

$$
\beta_{Q} \int_{0}^{\tau_{*}} \int_{\Omega}\left(\phi_{*}-\phi_{Q}\right) \xi \mathrm{d} x \mathrm{~d} t+\beta_{\Omega} \int_{\Omega}\left(\phi_{*}\left(\tau_{*}\right)-\phi_{\Omega}\right) \xi\left(\tau_{*}\right) \mathrm{d} x+\alpha_{Q} \int_{0}^{\tau_{*}} \int_{\Omega}\left(\sigma_{*}-\sigma_{Q}\right) \rho \mathrm{d} x \mathrm{~d} t
$$




$$
+\frac{\beta_{S}}{2} \int_{\Omega} \xi\left(\tau_{*}\right) \mathrm{d} x+\beta_{u} \int_{0}^{T} \int_{\Omega} u_{*}\left(u-u_{*}\right) \mathrm{d} x \mathrm{~d} t \geq 0, \quad \forall u \in \mathcal{U}_{\mathrm{ad}},
$$

where $(\xi, \eta, \rho)$ is the unique solution to the linearized problem (5.2)-(5.6) with $h=u-u_{*}$. Besides, setting

$$
\begin{aligned}
\mathcal{L}\left(\phi_{*}, \sigma_{*}, \tau_{*}\right)= & \frac{\beta_{Q}}{2} \int_{\Omega}\left|\phi_{*}\left(\tau_{*}\right)-\phi_{Q}\left(\tau_{*}\right)\right|^{2} \mathrm{~d} x+\beta_{\Omega} \int_{\Omega}\left(\phi_{*}\left(\tau_{*}\right)-\phi_{\Omega}\right) \partial_{t} \phi_{*}\left(\tau_{*}\right) \mathrm{d} x \\
& +\frac{\alpha_{Q}}{2} \int_{\Omega}\left|\sigma_{*}\left(\tau_{*}\right)-\sigma_{Q}\right|^{2} \mathrm{~d} x+\frac{\beta_{S}}{2} \int_{\Omega} \partial_{t} \phi_{*}\left(\tau_{*}\right) \mathrm{d} x+\beta_{T}
\end{aligned}
$$

we have

$$
\mathcal{L}\left(\phi_{*}, \sigma_{*}, \tau_{*}\right) \begin{cases}\geq 0, & \text { if } \tau_{*}=0, \\ =0, & \text { if } \tau_{*} \in(0, T), \\ \leq 0, & \text { if } \tau_{*}=T .\end{cases}
$$

Proof. Recalling that $\mathcal{U}_{\mathrm{ad}}$ is a closed and convex subset of $L^{2}(Q)$, we can conclude (5.10) from standard arguments (with no need to be repeated here). On the other hand, we have $D_{\tau} \widetilde{\mathcal{J}}\left(u_{*}, \tau_{*}\right)\left(s-\tau_{*}\right) \geq 0$ for $s \in[0, T]$. Noting that in Theorem 5.1 we cannot exclude the trivial cases where $\tau_{*}=0$ or $\tau_{*}=T$, then we arrive at the conclusion (5.11).

The proof is complete.

It is possible to eliminate the variables $\xi$ and $\rho$ from the inequality (5.10). Suppose that $\left(u_{*}, \tau_{*}\right) \in \mathcal{U}_{\text {ad }} \times[0, T]$ is solution to the control problem $(\mathbf{C P})$ with associated state $\left(\phi_{*}, \mu_{*}, \sigma_{*}\right)=$ $\mathcal{S}\left(u_{*}\right)$. By using the formal Lagrangian method (see e.g., [55]), we introduce the following adjoint system (see [15] for a simplified case with $\beta_{S}=\beta_{T}=\alpha_{Q}=0$ ):

$$
\begin{array}{lc}
-\partial_{t} p+\Delta q-F^{\prime \prime}\left(\phi_{*}\right) q+P^{\prime}\left(\phi_{*}\right)\left(\sigma_{*}-\mu_{*}\right)(r-p)=\beta_{Q}\left(\phi_{*}-\phi_{Q}\right), & \text { in } Q, \\
q-\Delta p+P\left(\phi_{*}\right)(p-r)=0, & \text { in } Q, \\
-\partial_{t} r-\Delta r+P\left(\phi_{*}\right)(r-p)=\alpha_{Q}\left(\sigma_{*}-\sigma_{Q}\right), & \text { in } Q, \\
\partial_{\mathbf{n}} p=\partial_{\mathbf{n}} q=\partial_{\mathbf{n}} r=0, & \text { on } \Sigma, \\
r\left(\tau_{*}\right)=0, \quad p\left(\tau_{*}\right)=\beta_{\Omega}\left(\phi_{*}\left(\tau_{*}\right)-\phi_{\Omega}\right)+\frac{\beta_{S}}{2}, & \text { in } \Omega .
\end{array}
$$

We call $(p, q, r)$ a solution to the adjoint system (5.12) - (5.16) if and only if the triplet $(p, q, r)$ satisfies the following conditions:

$$
\begin{aligned}
& p \in H^{1}\left(0, T ;\left(H_{N}^{2}(\Omega)\right)^{\prime}\right) \cap C^{0}\left([0, T] ; L^{2}(\Omega)\right) \cap L^{2}\left(0, T ; H_{N}^{2}(\Omega)\right), \\
& q \in L^{2}(Q), \\
& r \in H^{1}\left(0, T ; L^{2}(\Omega)\right) \cap C^{0}\left([0, T] ; H^{1}(\Omega)\right) \cap L^{2}\left(0, T ; H_{N}^{2}(\Omega)\right) .
\end{aligned}
$$

The equations (5.13) $-(5.16)$ are satisfied almost everywhere in their respective domains, however, since the final value $p\left(\tau_{*}\right)$ only belongs to $L^{2}(\Omega)$ (see $(\mathbf{C} 2)$ ), the regularity of $p$ is quite low so that (5.12) and the related boundary condition in (5.15) have to be understood in the weak variational sense, namely

$$
\begin{aligned}
& \left\langle-\partial_{t} p(t), v\right\rangle_{\left(H_{N}^{2}(\Omega)\right)^{\prime}, H_{N}^{2}(\Omega)}+\int_{\Omega} q(t) \Delta v \mathrm{~d} x-\int_{\Omega} F^{\prime \prime}\left(\phi_{*}(t)\right) q(t) v \mathrm{~d} x \\
& \quad+\int_{\Omega} P^{\prime}\left(\phi_{*}(t)\right)\left(\sigma_{*}(t)-\mu_{*}(t)\right)(r(t)-p(t)) v \mathrm{~d} x=\int_{\Omega} \beta_{Q}\left(\phi_{*}(t)-\phi_{Q}(t)\right) v \mathrm{~d} x,
\end{aligned}
$$


for all $v \in H_{N}^{2}(\Omega)$ and almost every $t \in(0, T)$.

Let us notice that the only difference between the adjoint system (5.12)-(5.16) and the one of [15] consists in the presence of a non-zero right hand side in equation (5.14) and a constant $\beta_{S}$ in (5.16). Since this extra right-hand side is indeed an $L^{2}(Q)$-function, we can repeat exactly the same argument used in [15, Theorem 4.2] to obtain the following existence and uniqueness result:

Proposition 5.5. Assume that (P1), (F1), (U1), (C1)-(C3) are satisfied, $\phi_{0} \in H_{N}^{2}(\Omega) \cap$ $H^{3}(\Omega)$, and $\sigma_{0} \in H^{1}(\Omega)$. Then the adjoint system (5.12)-(5.16) has a unique weak solution $(p, q, r)$ on $[0, T]$ in the sense formulated above.

Finally, we are able to rewrite the first order necessary optimality condition (5.10) for the minimizer $\left(u_{*}, \tau_{*}\right)$ using the adjoint states:

Corollary 5.1 (First order necessary optimality condition via adjoint states). Assume that the assumptions of Theorem 5.2 are satisfied. Let $\left(u_{*}, \tau_{*}\right) \in \mathcal{U}_{\mathrm{ad}} \times[0, T]$ denote a minimizer to the optimal control problem (CP) with corresponding state variables $\left(\phi_{*}, \mu_{*}, \sigma_{*}\right)=\mathcal{S}\left(u_{*}\right)$ and associated adjoint variables $(p, q, r)$. Then, the variational inequality (5.10) can be written as

$$
\beta_{u} \int_{0}^{T} \int_{\Omega} u_{*}\left(u-u_{*}\right) \mathrm{d} x \mathrm{~d} t+\int_{0}^{\tau_{*}} \int_{\Omega} r\left(u-u_{*}\right) \mathrm{d} x \mathrm{~d} t \geq 0, \quad \forall u \in \mathcal{U}_{\mathrm{ad}} .
$$

Remark 5.2. For the proof we can refer to [15, Theorem 4.3] with slight modifications due to the presence of the term $\alpha_{Q}\left(\sigma_{*}-\sigma_{Q}\right)$ in equation (5.14). Besides, if we extend $r$ by zero to $\left(\tau_{*}, T\right]$, then we can express (5.17) as

$$
\int_{0}^{T} \int_{\Omega}\left(\beta_{u} u_{*}+r\right)\left(u-u_{*}\right) \mathrm{d} x \mathrm{~d} t \geq 0, \quad \forall u \in \mathcal{U}_{\mathrm{ad}},
$$

which allows the interpretation that the optimal control $u_{*}$ is indeed the $L^{2}(Q)$-projection of $-\beta_{u}^{-1}$ r onto the set $\mathcal{U}_{\mathrm{ad}}$ (provided that $\beta_{u}>0$ ).

\section{Appendix}

Let $m \in \mathbb{R}$ be a given constant. We consider the following nonlocal elliptic boundary value problem

$$
\begin{cases}-\Delta \phi+F^{\prime}(\phi)=m-|\Omega|^{-1} \int_{\Omega} \phi \mathrm{d} x, & \text { in } \Omega, \\ \partial_{\nu} \phi=0, & \text { on } \partial \Omega .\end{cases}
$$

Problem (6.1) can be associated with the following functional

$$
\Upsilon(\phi)=\int_{\Omega}\left(\frac{1}{2}|\nabla \phi|^{2}+F(\phi)\right) \mathrm{d} x+\frac{1}{2}|\Omega|\left(m-|\Omega|^{-1} \int_{\Omega} \phi \mathrm{d} x\right)^{2}, \quad \forall \phi \in H^{1}(\Omega) .
$$

The following result has been obtained in [60, Lemma 3.1, Lemma 3.2].

Lemma 6.1. Let assumption (F1) be satisfied.

(1) Suppose that $\psi \in H_{N}^{2}(\Omega)$ is a (strong) solution to problem (6.1). Then $\psi$ is a critical point of the functional $\Upsilon(\phi)$ in $H^{1}(\Omega)$. Conversely, if $\psi$ is a critical point of the functional $\Upsilon(\phi)$ in $H^{1}(\Omega)$, then $\psi \in H_{N}^{2}(\Omega)$ and it is a strong solution to problem (6.1).

(2) The functional $\Upsilon(\phi)$ has at least one minimizer $\psi \in H^{1}(\Omega)$ such that

$$
\Upsilon(\psi)=\inf _{\phi \in H^{1}(\Omega)} \Upsilon(\phi) .
$$


Remark 6.1. By the elliptic regularity theory and a bootstrap argument, the minimizer $\psi$ is indeed a classical solution such that $\psi \in C^{\infty}(\bar{\Omega})$, provided that the boundary $\partial \Omega$ is smooth.

Associated with problem (6.1), the following Łojasiewicz-Simon type inequality has been proven in [60, Lemma 4.1] (see [62, Lemma 2.2] for a slightly weaker version).

Lemma 6.2. Let $(\boldsymbol{F} 1)$ and $(\boldsymbol{F} 2)$ be satisfied. Suppose that $\psi$ is a critical point of $\Upsilon(\phi)$ in $H^{1}(\Omega)$. Then there exist constants $\theta \in\left(0, \frac{1}{2}\right)$ and $\beta>0$, depending on $\psi, m$ and $\Omega$, such that, for any $\phi \in H^{1}(\Omega)$ with $\|\phi-\psi\|_{H^{1}(\Omega)}<\beta$, it holds

$$
\left\|-\Delta \phi+F^{\prime}(\phi)-\left(m-|\Omega|^{-1} \int_{\Omega} \phi \mathrm{d} x\right)\right\|_{\left(H^{1}(\Omega)\right)^{\prime}} \geq|\Upsilon(\phi)-\Upsilon(\psi)|^{1-\theta} .
$$

Now we are in a position to prove the Łojasiewicz-Simon type inequality (3.38) stated in Lemma 3.1, which plays a crucial role in the study of long-time behavior of problem (1.1)-(1.5).

Proof of Lemma 3.1. In Lemma 6.2, we take $m=m_{\infty}$ (see (3.35)) and $\psi=\phi_{\infty}$. Then it follows from (3.3)-(3.5) and (3.35) that $\phi_{\infty}$ satisfies the reduced elliptic problem (6.1). Hence, according to Lemma 6.1, we see that it is a critical point of $\Upsilon(\phi)$ (cf. (6.2) with $m=m_{\infty}$ ). As a consequence, Lemma 6.2 applies with constants $\theta \in\left(0, \frac{1}{2}\right), \beta>0$ depending on $\phi_{\infty}, m_{\infty}$ and $\Omega$. On the other hand, for any $\phi \in H_{N}^{2}(\Omega)$ we set

$$
\mu=-\Delta \phi+F^{\prime}(\phi)
$$

and then using integration by parts, we get

$$
\int_{\Omega} \mu \mathrm{d} x=\int_{\Omega} F^{\prime}(\phi) \mathrm{d} x .
$$

From the Łojasiewicz-Simon inequality (6.4) (applying to $\psi=\phi_{\infty}$ ), Poincaré's inequality and (3.37), we deduce that

$$
\begin{aligned}
\mid \Upsilon(\phi) & -\left.\Upsilon\left(\phi_{\infty}\right)\right|^{1-\theta} \\
\leq & \left\|-\Delta \phi+F^{\prime}(\phi)-\left(m_{\infty}-|\Omega|^{-1} \int_{\Omega} \phi \mathrm{d} x\right)\right\|_{\left(H^{1}(\Omega)\right)^{\prime}} \\
\leq & \left\|-\Delta \phi+F^{\prime}(\phi)-|\Omega|^{-1} \int_{\Omega} F^{\prime}(\phi) \mathrm{d} x\right\|_{\left(H^{1}(\Omega)\right)^{\prime}} \\
& +\left\||\Omega|^{-1} \int_{\Omega} F^{\prime}(\phi) \mathrm{d} x-\left(m_{\infty}-|\Omega|^{-1} \int_{\Omega} \phi \mathrm{d} x\right)\right\|_{\left(H^{1}(\Omega)\right)^{\prime}} \\
= & \|\mu-\bar{\mu}\|_{\left(H^{1}(\Omega)\right)^{\prime}}+\left\||\Omega|^{-1} \int_{\Omega} \mu \mathrm{d} x-|\Omega|^{-1} \int_{\Omega} \sigma \mathrm{d} x-m_{u}\right\|_{\left(H^{1}(\Omega)\right)^{\prime}} \\
\leq & \|\mu-\bar{\mu}\|_{\left(H^{1}(\Omega)\right)^{\prime}}+|\Omega|^{-1}\left|\int_{\Omega}(\mu-\sigma) \mathrm{d} x\right|^{+}\left|m_{u}\right| \\
\leq & \|\mu-\bar{\mu}\|_{\left(H^{1}(\Omega)\right)^{\prime}}+|\Omega|^{-1}\left(\int_{\Omega} \frac{1}{P(\phi)} \mathrm{d} x\right)^{\frac{1}{2}}\left(\int_{\Omega} P(\phi)(\mu-\sigma)^{2} \mathrm{~d} x\right)^{\frac{1}{2}} \\
& +\left|m_{u}\right| .
\end{aligned}
$$

By the Sobolev embedding $H^{2}(\Omega) \hookrightarrow C(\bar{\Omega})(n=2,3)$, the continuity as well as the strictly positivity of $P(s)$, then it holds

$$
\int_{\Omega} \frac{1}{P(\phi)} \mathrm{d} x \leq|\Omega|\left(\min _{x \in \bar{\Omega}} P(\phi(x))\right)^{-1} \leq C,
$$


where the constant $C>0$ depends on $\Omega,\|\phi\|_{H^{2}(\Omega)}$ and $P$.

On the other hand, on account of (1.6), (3.37), (6.2) and Poincaré's inequality, since $\theta \in\left(0, \frac{1}{2}\right)$ and $\sigma \in H^{1}(\Omega)$, we infer that

$$
\begin{aligned}
|\mathcal{E}(\phi, \sigma)-\Upsilon(\phi)|^{1-\theta} & \\
& =\left|\frac{1}{2}\|\sigma\|_{L^{2}(\Omega)}^{2}-\frac{1}{2}\right| \Omega\left|\left(\bar{\sigma}+m_{u}\right)^{2}\right|^{1-\theta} \\
& \leq\left(\frac{1}{2}\right)^{1-\theta}\left(\int_{\Omega}(\sigma-\bar{\sigma})^{2} \mathrm{~d} x+2|\Omega||\bar{\sigma}|\left|m_{u}\right|+|\Omega| m_{u}^{2}\right)^{1-\theta} \\
& \leq C\|\nabla \sigma\|_{L^{2}(\Omega)}^{2(1-\theta)}+C\left(\left|m_{u}\right|^{1-\theta}+\left|m_{u}\right|^{2(1-\theta)}\right) \\
& \leq C\|\nabla \sigma\|_{L^{2}(\Omega)}+C\left|m_{u}\right|^{\frac{1}{2}} .
\end{aligned}
$$

Finally, since $\Upsilon\left(\phi_{\infty}\right)=\mathcal{E}\left(\phi_{\infty}, \sigma_{\infty}\right)$ (recalling that $\sigma_{\infty}$ is a constant satisfying (3.37)), we deduce from inequalities (6.5) and (6.6) that

$$
\begin{aligned}
& \left|\mathcal{E}(\phi, \sigma)-\mathcal{E}\left(\phi_{\infty}, \sigma_{\infty}\right)\right|^{1-\theta} \\
& \quad \leq|\mathcal{E}(\phi, \sigma)-\Upsilon(\phi)|^{1-\theta}+\left|\Upsilon(\phi)-\Upsilon\left(\phi_{\infty}\right)\right|^{1-\theta} \\
& \quad \leq\|\mu-\bar{\mu}\|_{\left(H^{1}(\Omega)\right)^{\prime}}+C\|\nabla \sigma\|_{L^{2}(\Omega)}+C\|\sqrt{P(\phi)}(\mu-\sigma)\|_{L^{2}(\Omega)}+C\left|m_{u}\right|^{\frac{1}{2}} .
\end{aligned}
$$

The proof of Lemma 3.1 is complete.

\section{Acknowledgements}

This research has been performed in the framework of the project Fondazione Cariplo-Regione Lombardia MEGAsTAR "Matematica d'Eccellenza in biologia ed ingegneria come acceleratore di una nuova strateGia per l'ATtRattività dell'ateneo pavese". C. Cavaterra and E. Rocca were partially supported by GNAMPA (Gruppo Nazionale per l'Analisi Matematica, la Probabilità e le loro Applicazioni) of INdAM (Istituto Nazionale di Alta Matematica). H. Wu was partially supported by NNSFC grant No. 11631011 and the Shanghai Center for Mathematical Sciences. This research was also supported by the Italian Ministry of Education, University and Research (MIUR): Dipartimenti di Eccellenza Program (2018-2022) - Dept. of Mathematics "F. Casorati", University of Pavia.

\section{References}

[1] A. Agosti, P.-F. Antonietti, P. Ciarletta, M. Grasselli and M. Verani, A Cahn-Hilliard-type equation with application to tumor growth dynamics, Math. Methods Appl. Sci., 40 (2017), 7598-7626.

[2] R.-P. Araujo, D.L.S. McElwain, A History of the Study of Solid Tumour Growth: The Contribution of Mathematical Modelling, Bull. Math. Biol. 66 (2004), 1039-1091.

[3] R. Bellman, Stability Theory of Differential Equations, New York, McGraw-Hill Book Co., Inc., 1953.

[4] I. Ben Hassen, Decay estimates to equilibrium for some asymptotically autonomous semilinear evolution equations, Asymptot. Anal., 69(1\&2) (2010), 31-44.

[5] S. Bosia, M. Conti, M. Grasselli, On the Cahn-Hilliard-Brinkman system, Commun. Math. Sci., 13 (2015), 1541-1567. 
[6] C. Cavaterra, E. Rocca and H. Wu, Optimal boundary control of a simplified Ericksen-Leslie system for nematic liquid crystal flows in 2D, Arch. Ration. Mech. Anal., 224(3) (2017), $1037-1086$.

[7] J.-W. Cahn and J.-E. Hilliard, Free energy of a nonuniform system. I. Interfacial free energy, J. Chem. Phys., 28 (1958), 258-267.

[8] R. Chill and M.-A. Jendoubi, Convergence to steady states in asymptotically autonomous semilinear evolution equations, Nonlinear Anal., 53 (2003), 1017-1039.

[9] P. Colli, M.-H. Farshbaf-Shaker, G. Gilardi and J. Sprekels, Optimal boundary control of a viscous Cahn-Hilliard system with dynamic boundary condition and double obstacle potentials, SIAM J. Control Optim., 53 (2015), 2696-2721.

[10] P. Colli, M.-H. Farshbaf-Shaker, G. Gilardi and J. Sprekels, Second-order analysis of a boundary control problem for the viscous Cahn-Hilliard equation with dynamic boundary conditions, Ann. Acad. Rom. Sci. Math. Appl., 7 (2015), 41-66.

[11] P. Colli, G. Gilardi and D. Hilhorst, On a Cahn-Hilliard type phase field model related to tumor growth, Discrete Contin. Dyn. Syst., 35 (2015), 2423-2442.

[12] P. Colli, G. Gilardi, G. Marinoschi and E. Rocca. Sliding mode control for phase field system related to tumor growth, Appl. Math. Optim., (2017), DOI: 10.1007/s00245-017-9451-z.

[13] P. Colli, G. Gilardi, E. Rocca and J. Sprekels, Vanishing viscosities and error estimate for a Cahn-Hilliard type phase-field system related to tumor growth, Nonlinear Anal. Real World Appl., 26 (2015), 93-108.

[14] P. Colli, G. Gilardi, E. Rocca and J. Sprekels, Asymptotic analyses and error estimates for a Cahn-Hilliard type phase field system modelling tumor growth, Discrete Contin. Dyn. Syst. Ser. S., 10 (2017), 37-54.

[15] P. Colli, G. Gilardi, E. Rocca and J. Sprekels, Optimal distributed control of a diffuse interface model of tumor growth, Nonlinearity, 30 (2017), 2518-2546.

[16] P. Colli, G. Gilardi and J. Sprekels, A boundary control problem for the viscous CahnHilliard equation with dynamic boundary conditions, Appl. Math. Optim., 73 (2016), 195225 .

[17] P. Colli, G. Gilardi and J. Sprekels, A boundary control problem for the pure Cahn-Hilliard equation with dynamic boundary conditions, Adv. Nonlinear Anal., 4 (2015), 311-325.

[18] P. Colli and J. Sprekels, Optimal control of an Allen-Cahn equation with singular potentials and dynamic boundary condition, SIAM J. Control Optim., 53(1) (2015), 213-234.

[19] V. Cristini and J.-S. Lowengrub, Multiscale Modeling of Cancer. An Integrated Experimental and Mathematical Modeling Approach, Cambridge Univ. Press, 2010.

[20] V. Cristini, X. Li, J.-S. Lowengrub and S.-M. Wise, Nonlinear simulations of solid tumor growth using a mixture model: invasion and branching, J. Math. Biol., 58 (2009), 723-763.

[21] M.-M. Dai, E. Feireisl, E. Rocca, G. Schimperna and M. Schonbek, Analysis of a diffuse interface model for multispecies tumor growth, Nonlinearity, 30 (2017), 1639-1658.

[22] M. Ebenbeck and H. Garcke, On a Cahn-Hilliard-Brinkman model for tumour growth and its singular limits, preprint arXiv:1811.06699v1 (2018).

[23] E. Feireisl and F. Simondon, Convergence for semilinear degenerate parabolic equations in several space dimensions, J. Dynam. Differential Equations, 12(3) (2000), 647-673.

[24] S. Frigeri, M. Grasselli and E. Rocca, On a diffuse interface model of tumor growth, European J. Appl. Math., 26 (2015), 215-243. 
[25] S. Frigeri, K.-F. Lam, E. Rocca and G. Schimperna, On a multi-species Cahn-Hilliard-Darcy tumor growth model with singular potentials, Comm Math Sci., 16 (2018), 821-856.

[26] S. Frigeri, E. Rocca and J. Sprekels, Optimal distributed control of a nonlocal CahnHilliard/Navier-Stokes system in 2D, SIAM J. Control Optim., 54 (2016), 221-250.

[27] C.-G. Gal and H. Wu, Asymptotic behavior of a Cahn-Hilliard equation with Wentzell boundary conditions and mass conservation, Discrete Contin. Dyn. Syst., 22 (2008), 10411063.

[28] H. Garcke and K.-F. Lam, Global weak solutions and asymptotic limits of a Cahn-HilliardDarcy system modelling tumour growth, AIMS Mathematics, 1 (2016), 318-360.

[29] H. Garcke and K.-F. Lam, Well-posedness of a Cahn-Hilliard system modelling tumour growth with chemotaxis and active transport, European J. Appl. Math., 28 (2017), 284316.

[30] H. Garcke, K.-F. Lam, R. Nürnberg and E. Sitka, A multiphase Cahn-Hilliard-Darcy model for tumour growth with necrosis, Math. Models Methods Appl. Sci., 28 (2018), 525-577.

[31] H. Garcke, K.-F. Lam and E. Rocca, Optimal control of treatment time in a diffuse interface model of tumor growth, Appl. Math. Optim., (2017), DOI: 10.1007/s00245-017-9414-4.

[32] H. Garcke, K.-F. Lam, E. Sitka and V. Styles, A Cahn-Hilliard-Darcy model for tumour growth with chemotaxis and active transport, Math. Models Methods Appl. Sci. 26 (2016), 1095-1148.

[33] A. Giorgini, M. Grasselli and H. Wu, On the Cahn-Hilliard-Hele-Shaw system with singular potential, Ann. Inst. H. Poincare Anal. Non Lineaire, 35(4) (2018), 1079-1118.

[34] M. Grasselli, H. Wu and S. Zheng, Asymptotic behavior of a non-isothermal GinzburgLandau model, Quart. Appl. Math., 66(4) (2008), 743-770.

[35] A. Hawkins-Daarud, K.-G. van der Zee and J.-T. Oden, Numerical simulation of a thermodynamically consistent four-species tumor growth model, Int. J. Numer. Meth. Biomed. Engng., 28 (2011), 3-24.

[36] A. Hawkins-Daarud, S. Prudhomme, K.G. van der Zee and J.-T. Oden, Bayesian calibration, validation, and uncertainty quantification of diffuse interface models of tumor growth, J. Math. Biol., 67 (2013), 1457-1485.

[37] D. Hilhorst, J. Kampmann, T.-N. Nguyen and K.-G. van der Zee, Formal asymptotic limit of a diffuse-interface tumor-growth model, Math. Models Methods Appl. Sci., 25 (2015), $1011-1043$.

[38] M. Hintermüller and D. Wegner, Distributed optimal control of the Cahn-Hilliard system including the case of a double-obstacle homogeneous free energy density, SIAM J. Control Optim., 50 (2012), 388-418.

[39] M. Hintermüller and D. Wegner, Optimal control of a semidiscrete Cahn-Hilliard-NavierStokes system, SIAM J. Control Optim., 52 (2014), 747-772.

[40] S.-Z. Huang and P. Takáč, Convergence in gradient-like systems which are asymptotically autonomous and analytic, Nonlinear Anal., 46 (2001), 675-698.

[41] J. Jiang, H. Wu and S. Zheng, Well-posedness and long-time behavior of a non-autonomous Cahn-Hilliard-Darcy system with mass source modeling tumor growth, J. Differential Equations, 259 (2015), 3032-3077.

[42] C. Liu and H. Wu, An energetic variational approach for the Cahn-Hilliard equation with dynamic boundary condition: model derivation and mathematical analysis, Arch. Rational Mech. Anal., to appear, 2019, DOI: 10.1007/s00205-019-01356-x. 
[43] J.-S. Lowengrub, H.-B. Frieboes, F. Jin, Y.-L. Chuang, X. Li, P. Macklin, S.-M. Wise and V. Cristini, Nonlinear modelling of cancer: Bridging the gap between cells and tumours, Nonlinearity, 23(1) (2010), R1-R91.

[44] J.-S. Lowengrub, E. Titi and K. Zhao, Analysis of a mixture model of tumor growth, European J. Appl. Math., 24 (2013), 691-734.

[45] S. Melchionna and E. Rocca, Varifold solutions of a sharp interface limit of a diffuse interface model for tumor growth, Interfaces and Free Bound., 19 (2018), 571-590.

[46] A. Miranville, Asymptotic behavior of the Cahn-Hilliard-Oono equation, J. Appl. Anal. Comput., 1 (2011), 523-536.

[47] A. Miranville, E. Rocca, and G. Schimperna, On the long time behavior of a tumor growth model, preprint arXiv:1810.12239 (2018), 1-19.

[48] J.-T. Oden, A. Hawkins and S. Prudhomme, General diffuse-interface theories and an approach to predictive tumor growth modeling, Math. Models Methods Appl. Sci., 20(3) (2010), 477-517.

[49] J.-T. Oden, E.-E. Prudencio and A. Hawkins-Daarud, Selection and assessment of phenomenological models of tumor growth, Math. Models Methods Appl. Sci., 23(7) (2013), 1309-1338.

[50] E. Rocca and R. Scala, A rigorous sharp interface limit of a diffuse interface model related to tumor growth, J. Nonlinear Sci., 27 (2017), 847-872.

[51] E. Rocca and J. Sprekels, Optimal distributed control of a nonlocal convective Cahn-Hilliard equation by the velocity in three dimensions, SIAM J. Control Optim., 53 (2015), 16541680 .

[52] P. Rybka and K.-H. Hoffmann, Convergence of solutions to Cahn-Hilliard equation, Commun. Partial Differential Equations, 24(5\&6) (1999), 1055-1077.

[53] L. Simon, Asymptotics for a class of nonlinear evolution equation with applications to geometric problems, Ann. of Math., 118 (1983), 525-571.

[54] J. Sprekels and H. Wu, Optimal distributed control of a Cahn-Hilliard-Darcy system with mass sources, Appl. Math. Optim., to appear, 2019. DOI: 10.1007/s00245-019-09555-4

[55] F. Tröltzsch, Optimal Control of Partial Differential Equations. Theory, Methods and Applications, Grad. Stud. in Math., Vol. 112, AMS, Providence, RI, 2010.

[56] X.-M. Wang and H. Wu, Long-time behavior for the Hele-Shaw-Cahn-Hilliard system, Asymptot. Anal., 78(4) (2012), 217-245.

[57] X.-M. Wang and Z.-F. Zhang, Well-posedness of the Hele-Shaw-Cahn-Hilliard system, Ann. Inst. H. Poincaré Anal. Non Linéaire, 30(3) (2013), 367-384.

[58] X. Wu, G.-J. van Zwieten and K.-G. van der Zee, Stabilized second-order convex splitting schemes for Cahn-Hilliard models with applications to diffuse-interface tumor-growth models, Int. J. Numer. Meth. Biomed. Engng., 30 (2014), 180-203.

[59] H. Wu, Convergence to equilibrium for a Cahn-Hilliard model with the Wentzell boundary condition, Asymptotic Anal., 54(1\&2) (2007), 71-92.

[60] H. Wu, M. Grasselli and S. Zheng, Convergence to equilibrium for a parabolic-hyperbolic phase-field system with Neumann boundary conditions, Math. Models Methods Appl. Sci., 17(1) (2007), 125-153.

[61] H. Wu and S. Zheng, Convergence to equilibrium for the Cahn-Hilliard equation with dynamic boundary conditions, J. Differential Equations, 204(2) (2004), 511-531. 
[62] Z. Zhang, Asymptotic behavior of solutions to the phase-field equations with Neumann boundary conditions, Commun. Pure Appl. Anal., 4(3) (2005), 683-693.

[63] X. Zhao and C. Liu, Optimal control of the convective Cahn-Hilliard equation, Appl. Anal., 92 (2013), 1028-1045.

[64] X. Zhao and C. Liu, Optimal control for the convective Cahn-Hilliard equation in 2D case, Appl. Math. Optim., 70 (2014), 61-82.

[65] S. Zheng, Nonlinear Evolution Equations, Pitman series Monographs and Survey in Pure and Applied Mathematics, 133, Chapman \& Hall/CRC, Boca Raton, 2004. 